\title{
Shock focusing in a planar convergent geometry: experiment and simulation
}

\author{
C. BOND, D. J. HILL†, D. I. MEIRON AND P. E. DIMOTAKIS
}

Graduate Aeronautical Laboratories, California Institute of Technology, Pasadena, CA 91125, USA

(Received 26 March 2008; revised 7 August 2009; accepted 8 August 2009; first published online 16 November 2009)

The behaviour of an initially planar shock wave propagating into a linearly convergent wedge is investigated experimentally and numerically. In the experiment, a $25^{\circ}$ internal wedge is mounted asymmetrically in a pressure-driven shock tube. Shock waves with incident Mach numbers in the ranges of 1.4-1.6 and 2.4-2.6 are generated in nitrogen and carbon dioxide. During each run, the full pressure history is recorded at fourteen locations along the wedge faces and schlieren images are produced. Numerical simulations performed based on the compressible Euler equations are validated against the experiment. The simulations are then used as an additional tool in the investigation.

The linearly convergent geometry strengthens the incoming shock repeatedly, as waves reflected from the wedge faces cross the interior of the wedge. This investigation shows that aspects of this structure persist through multiple reflections and influence the nature of the shock-wave focusing. The shock focusing resulting from the distributed reflected waves of the Mach 1.5 case is distinctly different from the stepwise focusing at the higher incoming shock Mach number. Further experiments using $\mathrm{CO}_{2}$ instead of $\mathrm{N}_{2}$ elucidate some relevant real-gas effects and suggest that the presence or absence of a weak leading shock on the distributed reflections is not a controlling factor for focusing.

Key words: flow structure interactions, gas dynamics, shock waves

\section{Introduction}

A shock travelling into a converging geometry experiences strengthening as the walls force focusing. Setchell, Storm \& Sturtevant (1972) performed such experiments in a conical converging geometry. For straight converging walls, this focusing can be compared with the ideal cases of cylindrical (two-dimensional) and spherical (three-dimensional) converging shock waves. While exact solutions do not exist, Guderley (1942) produced asymptotic similarity solutions (in the strong-shock limit) for converging cylindrical and spherical waves that exhibited a power-law increase in the shock Mach number. Whitham's shock dynamics (Whitham 1957) provide an accurate approximation to the converging shocks that is valid even well below the strong-shock limit.

Because the expected intense pressure at the centre of a converging cylindrical or spherical shock could be considerably reduced by imperfect focusing, the study of the stability of such configurations has attracted both theoretical and experimental attention. The approximate stability theory (Whitham 1957) indicates that converging 
symmetrical shocks are weakly unstable and predicts the development of triple points. Experimental attempts to create convergent shocks (Takayama, Kleine \& Gronig 1987; Watanabe \& Takayama 1992) generally result in converging polygonal structures similar to those investigated in the context of shock dynamics theory (Schwendeman \& Whitham 1987). Since this instability is generally triggered by upstream disturbances, the nature of these polygonal waves can often be traced to imperfections in the original axisymmetry of the experiment.

There is interest in the generation of initially smooth converging shocks and their interaction with other flow structures in many contexts (Dimotakis \& Samtaney 2006) as well as an aid to understanding processes such as sonoluminescence (Evans 1996) and the Richtmyer-Meshkov instability in a convergent geometry (Hosseini \& Takayama 2005). The production of cylindrical waves has been addressed in practice with an annular or obstructed shock tube (Perry \& Kantrowitz 1951) and theoretically for the case of a gas lens that can focus a planar shock into a segment of a circular cylinder (Dimotakis \& Samtaney 2006).

Another method to produce a smoothly focused wave is a log-spiral channel (Inoue, Takahashi \& Takayama 1993; Inoue et al. 1995), which converts a planar shock into a 90 degree arc of a cylindrical shock so gradually that no Mach reflections are produced at the walls. In this approach, slower but smoother acoustic reflections replace the Mach reflections in communicating the higher pressure to the shock front. However, since a linearly convergent channel of similar dimensions would focus in an angular segment with one-third to one-fourth the angle, this method produces weaker shocks for any given radius.

The experimentally confirmed instabilities of cylindrical waves led to the investigation of polygonal shock waves (Apazidis et al. 2002; Eliasson et al. 2006). These studies showed that the nature of the focusing depends, in part, on the nature of the reflected waves at the vertices of the polygons. For the triangular (three-sided) case, the reflections are regular, and the incoming shocks maintain a nearly constant strength until reaching the centre. For a larger number of sides, the reflections are Mach reflections, with the waves consuming the initial polygonal sides, replacing them in an alternating fashion with higher strength shocks. This results in increased shock strengths as the waves approach the centre, producing a stepwise focusing that mimics that of an ideal circular wave.

Shock focusing in a linearly convergent channel will resemble alternating polygonal waves and the incoming shock will be alternately strengthened by the repeated crossing of the Mach reflections across the channel height. The experiments of Setchell et al. (1972) with a conical geometry show that shock strength along the centreline increases stepwise and the focusing of the planar shock is caused by the interactions of the reflections off the sidewalls. However, such a geometry also produces additional focusing as the reflected waves strengthen as they converge radially on the axis. The two-dimensional case should show similar stepwise shock strengthening, but will not have the added focusing.

As shock strengthening is attributable to reflections of the incident wave from the converging walls, the nature of those reflections is of interest. If the flow produces simple Mach reflections in which the compression is contained entirely in a single reflected shock, the results would not merit new attention as the reflection of triple points from walls are well understood. However, at lower wedge angles and Mach numbers, the reflection structure is not characterized by this simple reflection regime. In particular, significant Mach stem curvature is observed that produces a distributed region of compression in the reflected wave. The current 
investigation compares shock focusing in flows with compact and distributed reflections; since weak reflections are subject to ongoing investigation and are less familiar than compact reflections, it is useful to review the literature on weak Mach reflections.

Ben-Dor (2006) provides an overview of the pseudo-steady Mach reflections, even though his characterization of relatively weak Mach reflections is limited. Colella \& Henderson (1990) considered some relatively weak Mach reflections and observed a distributed reflection, with no leading shock, in both experiment and computation, which they termed a von Neumann Reflection (vNR). They suggested a criterion for this type of reflection that involved the structure of the solution of the three-shock problem at the flow conditions. Others (Olim \& Dewey 1992; Sasoh \& Takayama 1994) also reported on this weak-reflection regime, but suggest that the triple-pointbased criterion of Colella \& Henderson (1990) was too broad. Sasoh \& Takayama (1994) suggest a different criterion based on a shock dynamics analysis that is more restrictive and better matches observed reflection structures.

Recent research (Sandeman 2000; Skews \& Ashworth 2005) has concentrated more on the behaviour of very weak incident shocks, and the structure very close to regions typically described as triple points is very interesting, but not in the scope of the present investigation. Additionally, Vasilev, Elperin \& Ben-Dor (2008) have recently provided a detailed analysis of three- and four-wave solutions at the triple point, given the triple-point trajectory and the incident shock Mach number. The fourwave solution structures typically consist of a leading reflected shock followed by an attached expansion fan; this might be expected to result in a lower pressure rise (and a Mach stem/an incident shock kink angle) than a simple reflected shock of the same Mach number. However, those studies are limited to the triple point and provide insufficient insight into the nature of distributed weak reflections. It is notable that this analysis used the term vNR to describe the triple-point structure, while Colella \& Henderson (1990) used the term to refer to a fully distributed reflection without a leading shock. This is unfortunate as some conditions that meet the criterion originally suggested by Colella \& Henderson (1990) may have weak leading shocks (instead of just acoustic waves) whose triple-point structure may fall into what is defined by Vasilev et al. (2008) as non-vNR wave patterns.

Because of the potential for confusion from the use of the term vNR, the current work accepts single Mach reflections as on a continuum between fully distributed (which is the structure Colella \& Henderson 1990 described) and fully compact (which would be a pure 3-shock structure with a completely straight Mach stem, which in practice is approached but not reached.)

The preceding studies assume an ideal-gas behaviour but the inclusion of real-gas effects on the triple-point structure is relatively straightforward for moderate and high shock Mach numbers (Ben-Dor 2007). These conditions have a common behaviour, typical of shocks in real gases: the shocks consist of a strong initial jump with a non-equilibrium relaxation zone following. However, this behaviour is not universal and low Mach number shocks may be more complicated. For example, Griffith \& Kenney (1957) found that in $\mathrm{CO}_{2}$ the shock jump becomes a dispersed supersonic compression wave for Mach numbers below $\approx 1.04$.

This paper studies the behaviour of a planar shock in a two-dimensional linearly convergent geometry to assess focusing and to better understand how the nature of the reflected waves affects the results. Specifically, this paper investigates how focusing associated with distributed reflections deviates from the idealized stepwise focusing expected from the compact strong Mach reflections. Experimental results 
are presented first, using $\mathrm{N}_{2}$ gas, with planar shocks sent into a converging geometry consisting of an asymmetric wedge, and later with $\mathrm{CO}_{2}$ gas to investigate the influence of real-gas effects.

The incident shock strengths chosen were at two conditions, within and outside of the distributed reflection regime seen by Colella \& Henderson (1990), so that the effect of the initial reflection structure on shock focusing could be elucidated. The $\mathrm{CO}_{2}$ runs were made at conditions similar to those of the $\mathrm{N}_{2}$ runs; real-gas effects were not expected to provide qualitative changes at the conditions in the $M \approx 2.5$ runs, but at the lower Mach number could produce a fully distributed reflected wave by replacing any weak leading shock with a fully dispersed supersonic compression wave. These two Mach number conditions are also, by comparison with the work of Henderson et al. (2003), known to exhibit differing behaviour of the slipline at the wall; the higher Mach number case will have the slipline roll up at the wall, while the lower Mach number case will not.

The numerical simulations carefully match the experimental conditions, with flow and geometric parameters such as temperature, incident Mach number and wedge angles providing the initial and boundary conditions for the simulations. A lowdissipation, high-resolution shock-capturing scheme (Hill \& Pullin 2004; Pantano et al. 2007) was used to compute the evolution of the flow. This is a hybrid method that uses a WENO flux interpolation at shocks and physical boundaries, but centred differences in a skew-symmetric form to compute fluxes in the remainder of the flow. Computational diagnostics are included in the simulations to record pressure histories at locations where the pressure transducers were located and enable a direct comparison with experiment.

Validated numerical simulations allowed a more detailed investigation of the flow. While experimental results were limited to wall pressures and single-time photographs, the computations provide the full flow information, allowing further interpretation of the experimental results. In particular, full flow pressure fields clarified the relation between the reflected wave structure and the observed wall-pressure traces.

The current investigation finds that the structure of the initial reflected waves persists even after those waves have reflected from the opposite walls; that the signature of that structure is readily discernible in the pressure traces measured; and that the smoother, distributed compression of the distributed reflections produce shock focusing closer to that of a cylindrical converging shock than the flow formed from an initial compact Mach reflection.

\section{Description of the experiment}

The discussion of the experimental results falls into two distinct parts. The first is an examination of the initial shock reflection and refraction process prior to any interaction between the shocks reflected off the upper and lower wedge plates. During this stage, the upper and lower Mach reflections can be viewed as independent events that share the same incoming shock speed but differ in the encountered wedge angle. The second part of the discussion follows the development of the shock structures as a whole. Of interest is shock focusing in the convergent geometry as can be seen by the full shock history in the experiment. This focusing is driven by the mutual interactions of the two primary reflections.

The conditions for the experiments were selected to investigate focusing associated with the initial reflection structure in a regime characterized by deviations from idealized three-shock structures, as described by Colella \& Henderson (1990). Care was taken to ensure that the magnified pressures obtained in the later stages of the 
Driver gases

$\mathrm{N} 1.5$

$\mathrm{N} 2.5$

C1.5

C2.5
$\mathrm{N}_{2}$

$\mathrm{He} / 5 \%$ air

$\mathrm{He} / 20 \%$ air

$\mathrm{He} / 5 \%$ air
Ambient pressure $(\mathrm{kPa})$

12

2.7

19.7

2.4
Incident Mach: $M_{i}$

1.5

2.5

1.5

2.5

TABLE 1. Nominal conditions for the different runs.

experiment due to the convergent focusing were still well within the design parameters of the facility. The wedge angles and the Mach numbers are also of interest in the context of lensed circular shock focusing (Dimotakis \& Samtaney 2006).

The choice of the converging wedge geometry provides, within a single experiment, both a significant shock interaction and a range of shock strengths. Reflected shock waves interact with each other and flow non-uniformities such as sliplines and distributed compression regions (as seen in the weak Mach reflections). While the incoming shock pressure jump is of the order of $20 \mathrm{kPa}$, the pressure rise from shock focusing can exceed $700 \mathrm{kPa}$, corresponding to a compression ratio of 35 .

The total wedge angle of $25^{\circ}$ provides a good compromise between the initial stages of shock reflection and later requirements for shock focusing. For the experiments presented here, an asymmetric configuration of the wedge was chosen to provide a stronger test for comparisons with simulations. Wedge angles of (approximately) $+10^{\circ}$ and $-15^{\circ}$ from the horizontal were chosen, respectively.

Table 1 describes the nominal flow conditions for this study. The main experiments used $\mathrm{N}_{2}$ as the test gas that was selected for its nearly ideal-gas behaviour under the conditions of the study. Further experiments were performed with $\mathrm{CO}_{2}$ as the test gas to assess the real-gas effects on shock focusing. The selection of the two Mach number ranges, 1.4-1.6 and 2.4-2.6, allowed an investigation of both weak Mach reflections where the reflection is substantially an extended compression zone involving curvature of the Mach stem (with, at best, a very weak leading reflected shock), and a stronger reflection where the compression is substantially associated with the reflected shock and the resultant kink at the triple point. In the latter case, the reflection would be close to the simple three-shock model of a Mach reflection and the focusing would be expected to occur in a stepwise fashion.

The experiments using $\mathrm{CO}_{2}$ are described in $\S 7$. It is noted that for the lower incident Mach number, the leading reflected wave will be below the $M_{\mathrm{s}} \approx 1.04$ cutoff for a fully dispersed supersonic compression wave (Griffith \& Kenney 1957) and no shock front is expected to form on the reflected wave.

In a standard shock tube, the incident shock is followed by an expansion wave that has reflected from the end of the driver section. The GALCIT 17-inch Shock Tube was originally optimized for shock Mach numbers significantly larger than those used in this study. For conditions similar to ones in the present investigation, Kumar, Hornung \& Sturtevant (2003) noted that an expansion wave immediately followed the incident shock in their experiments in this facility. They used a pure He driver gas to minimize the pressure ratio needed for a given shock Mach number and to allow a maximum test section pressure for a given diaphragm.

In the current study, the presence of the expansion wave in the test section was an undesirable complication to the interpretation of experimental results and the comparison with numerical simulation. For these experiments, either the use of $\mathrm{N}_{2}$ as the driver gas or the introduction of air into the driver section before finishing 


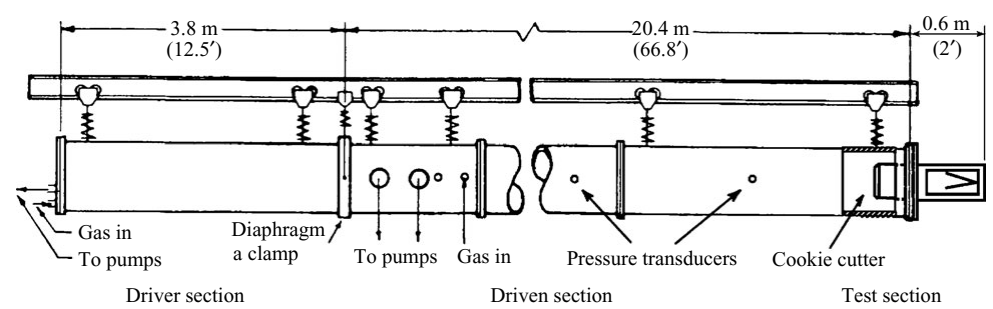

Figure 1. The GALCIT 17 inch Shock Tube.

the fill with He provided a simple method to delay the expansion wave arrival while retaining a relatively high test section pressure.

\section{Experimental apparatus and diagnostic system}

The GALCIT 17 inch Shock Tube is described by Liepmann et al. (1962). For this investigation, a rectangular test section designed and fabricated for the purpose was mounted at the end of the driven section of the main tube (figure 1). The main tube consists of a $43.2 \mathrm{~cm}$ diameter stainless steel tube, with a driver section of $3.8 \mathrm{~m}$ length and a driven section of $20.4 \mathrm{~m}$. The driver and driven sections are initially separated by a $0.25 \mathrm{~mm}$ thick aluminium diaphragm and consistent bursting (with typical repeatability to $\approx 1 \%$ in $M_{i}$ ) is ensured by a cruciform knife edge mounted in the tube on the driven side of the diaphragm. The discussion below documents the experimental and diagnostic details needed to assess the experimental data, with a particular emphasis on aspects that prove important in the successful comparison between experiment and simulation.

\subsection{Test section and apparatus}

The test section housing box was constructed specifically for these experiments with internal dimensions of $26.0 \mathrm{~cm} \times 15.2 \mathrm{~cm} \times 55.9 \mathrm{~cm}$. The sides of the box mount a pair of optical quality BK7 windows with dimensions $36.2 \mathrm{~cm} \times 15.9 \mathrm{~cm}$. The box itself is attached to the end of the shock tube with a flange that mounts a $30.5 \mathrm{~cm}$ long cookie cutter that separates a rectangular shock from the circular shock generated by the shock tube. Two pairs of pressure-transducer ports at the entrance of the test section allow for the measurement of arrival times and inference of the incoming shock speed. These transducer pairs are precision-located on opposing walls so that any tilt (i.e. deviation from vertical) of the incoming shock may be estimated.

The apparatus used in these experiments, shown in figure 2, is an internal wedge that forces the incoming shock to propagate into a converging geometry. The two plates, each of length of $30.5 \mathrm{~cm}$, are adjusted to approximately $+10^{\circ}$ and $-15^{\circ}$ from the horizontal, with the actual angles then measured to some precision, as discussed below. The apex of this wedge is terminated with the $6.4 \mathrm{~mm}$ radius hinge on the axis. This regularization was chosen in view of the accompanying numerical simulations and to limit the maximal pressures achieved. The wedge construction consists of a frame that holds the wedge axis and wedge supports in a fixed position relative to the test section. Two wedge plates are held by forks that are hinged at a fixed point in the test section. The tips of the plates have an included angle of $6^{\circ}$. Turnbuckles and screws are used to adjust the angle of the wedge plates with respect to the incoming shock. Wedge angles were set outside of the test section and measured against each other and the bottom support of the wedge using a digital level. The measurements were then compared with measurement of the distance between the wedge tips. In 


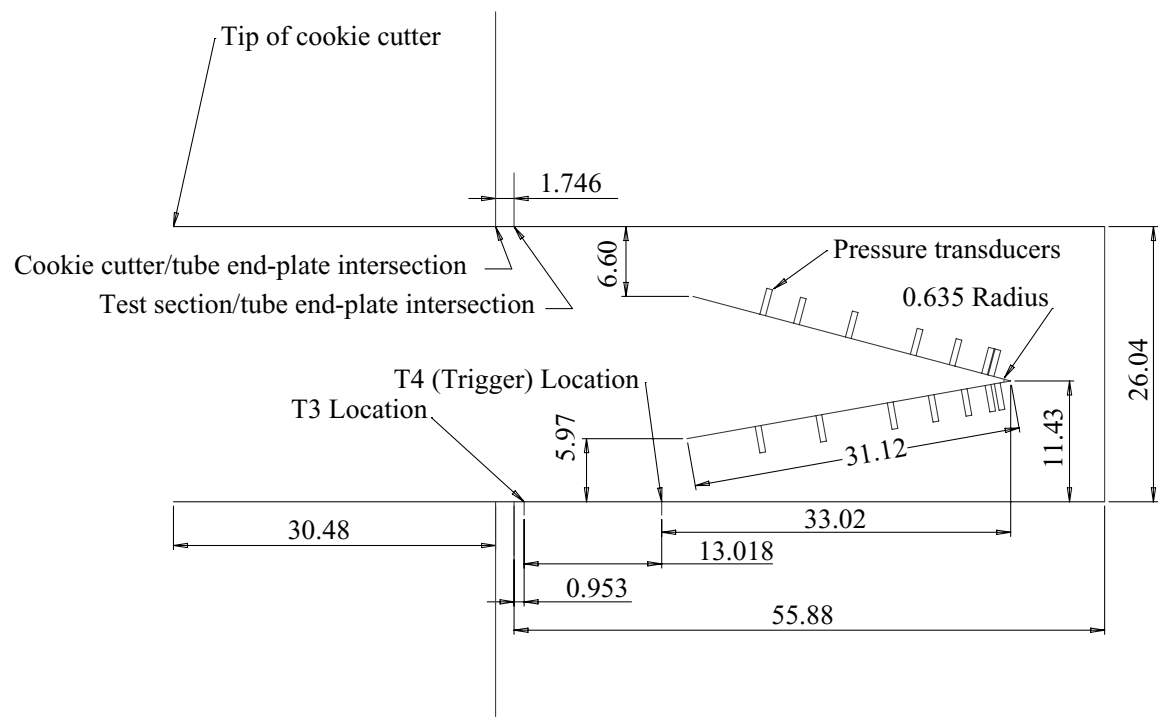

FiguRE 2. The geometry of the test section, including timing transducer locations. Wedge transducer widths and locations are shown to scale, with the locations indicated in table 2. The centres of the timing transducers (T3 and T4) are marked, while an additional timing transducer opposite T4 on the upper wall is omitted. All dimensions are in centimetres.

practice, the setting of the angles is less precise than the measurement; as the wedge was sometimes adjusted between runs, the wedge angles are not the same for all of the runs in this study.

The metal wedge is $14.7 \mathrm{~cm}$ wide, while the test section is $5 \mathrm{~mm}$ wider. The $2.5 \mathrm{~mm}$ space between the wedge and each of the optical windows is sealed with a $3 \mathrm{~mm}$ gasket. The thickness of the gasket made sealing at the wedge plate tips impractical; the gasket was typically terminated $2.5 \mathrm{~cm}$ short of the tips. Additionally, gasket compression tended to push the gasket into the field of view; to avoid this the gasket was trimmed, but the resulting gasket surface was no longer smooth. A second issue was that the wedge plates were butted up against the hinge at the apex with no provision for complete sealing.

The wedge itself is instrumented with fourteen pressure transducers, seven on each plate. These are mounted on the plate with Delrin inserts. Direct mounting of the transducers in the metal plate initially resulted in a high vibrational coupling between the plates and the transducer measurements that allowed the pressure field from the waves propagating through the steel plates to be registered, in part. The location of the pressure transducers on the plates and the test section is shown in figure 2, and the transducer locations on the wedge are tabulated in table 2.

\subsection{Pressure measurements}

The pressure measurements were made with piezoelectronic pressure transducers with integrated electronics manufactured by PCB Piezotronics. The pressure transducers were of two types: the basic Model 112A miniature high-sensitivity probes and the Model 113A frequency-tailored miniature probes. These probes shared a common form factor, with a $5.5 \mathrm{~mm}$ diameter sensing surface. The typical rise time for these transducers is $1-2 \mu \mathrm{s}$. The calibration of the transducers was performed by PCB Piezotronics. 


$\begin{array}{cccc}\text { Transducer } & \text { Distance from apex in cm } & \text { Transducer } & \text { Distance from a } \\ \text { L1 } & 24.3 & \mathrm{U} 1 & 24.3 \\ \text { L2 } & 18.4 & \mathrm{U} 2 & 21.0 \\ \text { L3 } & 11.4 & \mathrm{U} 3 & 15.9 \\ \text { L4 } & 7.62 & \mathrm{U} 4 & 9.52 \\ \text { L5 } & 4.45 & \mathrm{U} 5 & 5.71 \\ \text { L6 } & 2.16 & \mathrm{U} 6 & 2.54 \\ \text { L7 } & 1.27 & \mathrm{U} 7 & 1.91\end{array}$

TABLE 2. Pressure transducer locations on the wedge, as measured from the apex of the wedge. The inner radius of the wedge is at $6.4 \mathrm{~mm}$ from the apex, and the transducers are $5.5 \mathrm{~mm}$ in diameter. The L (lower) transducers are on the lower plate of the wedge, and the U (upper) transducers are on the upper plate of the wedge.

\begin{tabular}{|c|c|c|c|c|c|c|c|}
\hline \multirow[b]{2}{*}{ Run } & \multicolumn{2}{|c|}{ Driver } & \multirow{2}{*}{$\begin{array}{c}\text { Test section } \\
\qquad p(\mathrm{kPa})\end{array}$} & \multicolumn{4}{|c|}{ Measured parameters } \\
\hline & Gas & $p(\mathrm{kPa})$ & & $M_{i}$ & $\delta_{\mathrm{t}}(\mu \mathrm{s})$ & $t_{\text {picture }}(\mu \mathrm{s})$ & $T\left({ }^{\circ} \mathrm{C}\right)$ \\
\hline 68 & $\mathrm{~N}_{2}$ & 97 & 11.81 & 1.49 & 248.4 & 177.0 & 24.6 \\
\hline 69 & $\mathrm{~N}_{2}$ & 92 & 11.67 & 1.48 & 250.9 & 1053.0 & 24.2 \\
\hline 71 & $\mathrm{He}+5 \%$ air & 92 & 2.40 & 2.46 & 150.4 & 751.4 & 24.3 \\
\hline 72 & $\mathrm{He}+5 \%$ air & 87 & 2.40 & 2.43 & 152.4 & 151.1 & 23.9 \\
\hline
\end{tabular}

TABLE 3. Run conditions for individual $\mathrm{N}_{2}$ (test gas) runs.

These transducers have some inherent characteristics that affect the measurements. The transducers are high-pass with a cutoff frequency around $1 \mathrm{~Hz}$, requiring that pre-shock pressures must be measured separately before each run. Even after the improved transducer mounting noted above, there is still some coupling between wedge vibration and transducer signals. This coupling can most easily be seen in the experimental pressure traces before the first arrival of the shock. While the coupling is small, it is stronger towards the wedge tips. The transducers have resonant frequencies in the range of $300-500 \mathrm{kHz}$ that can result in minor signal ringing after the passage of a shock (greater in the $112 \mathrm{~A}$ probes than in the $113 \mathrm{~A}$ probes).

The pressure before the run is measured by a Druck DPI 260 pressure indicator with a built-in pressure transducer. This measurement is made shortly before the experiment and the gauge is isolated from the test section for the shot. This transducer/indicator pair was calibrated by Druck.

\subsection{Data acquisition}

The experimental data presented in this paper are of two types: pressure measurements and schlieren images. Pressure measurements from upstream were also used to trigger the data acquisition system and the schlieren spark timing.

The pressure measurements required high-speed data acquisition, which was done with both PC-based data acquisition boards and a digital oscilloscope. The digital oscilloscope was a LeCroy waveSurfer 424 used to acquire four channels digitised at 8 bits at $50 \mathrm{MHz}$ for $2 \mathrm{~ms}$. Three channels recorded the output of the shock timing pressure transducers; two marked as T3 and T4 in the test section diagram, figure 2, and the third, which is opposite T4 on the upper wall of the test section. The fourth channel was used to record the output of a high-speed photodiode (aimed at the schlieren spark source) to mark the actual time of the spark in the datarecording sequence. The PC-based data acquisition system consists of three National 
Instruments high-speed DAQ boards, with the data acquisition controlled within the NI LabView environment. Two separate NI 6110 boards were used and each of these provided four channels of 12 bit digitisation at $5 \mathrm{MHz}$. The third board, a NI PCI-6133, provided additional eight channels of 14 bit digitisation but at the lower rate of $2.5 \mathrm{MHz}$. All of the National Instruments boards use simultaneous sample and hold.

\subsection{Triggering}

The triggering of the pressure measurements and images originated from one of the inputs to the digital oscilloscope - that from the pressure transducer marked T4 (trigger) in figure 2. The oscilloscope trigger was also sent to a Berkeley Nucleonics Corporation Model 555 Pulse/Delay generator that directly triggered the PC DAQ boards while providing a delayed pulse to trigger the spark.

\subsection{Schlieren}

Images were recorded using the schlieren technique. The light source was a Xenon Corporation Nanopulser 437B, with a spark of roughly $20 \mathrm{~ns}$ duration. The optics were mounted in a folded Z-fold configuration on optical tables mounted at the test section height. The $45^{\circ}$ mirrors were of $25.4 \mathrm{~cm}$ diameter and folding was along the length of the test section so that the field of view was limited to a roughly $17 \mathrm{~cm}$ width while covering the entire height of the test section. Further details are discussed in Appendix B.

\section{Experimental results}

The experimental data consist of schlieren images centred on the entrance of the wedge taken as the shock enters, and pressure measurements on the wedge surfaces were continuously recorded throughout the run. The shock reflects separately from each wedge plate, initially producing two independent pseudo-steady Mach reflections. These pseudo-steady flows have two parts: the bow shock, which turns the previously shocked flow to accommodate the wedge plate, and the incident shock with a Mach stem that processes the quiescent gas.

The reflected waves subsequently interact with each other and reflect from the two wedge plates. The interaction of the reflected waves with the incoming shock results in a stepwise strengthening of the leading shock. When the incoming shock finally reflects off the small radius at the wedge apex, the shock is strengthened considerably, as expected. The returning shock then sweeps out of the wedge, consuming the previously reflected shocks and exiting the wedge as a nearly cylindrical divergent wave.

\subsection{Inflow photographs}

The location of the schlieren field of view at the entrance of the wedge is exploited with two distinct timings of images: that of incoming and outgoing shocks. The outgoing shock images are recorded after the converging experiment is complete. This later flow is discussed further in Appendix A. In the incident phase, the flow consists of two independent pseudo-steady Mach reflections.

Figure 3 shows the incident shock from Run 68, Mach 1.49 in $\mathrm{N}_{2}$ gas. The bow shocks stand clear of the wedge tips. Two Mach reflections are evident, with the slipline of the reflection off of the lower wedge $\left(9.8^{\circ}\right)$ barely visible. (The sliplines are more pronounced in figure 5, an oversaturated version of figure 3 included only for the purpose of exposing this feature.) Both sliplines are slightly curved towards the 


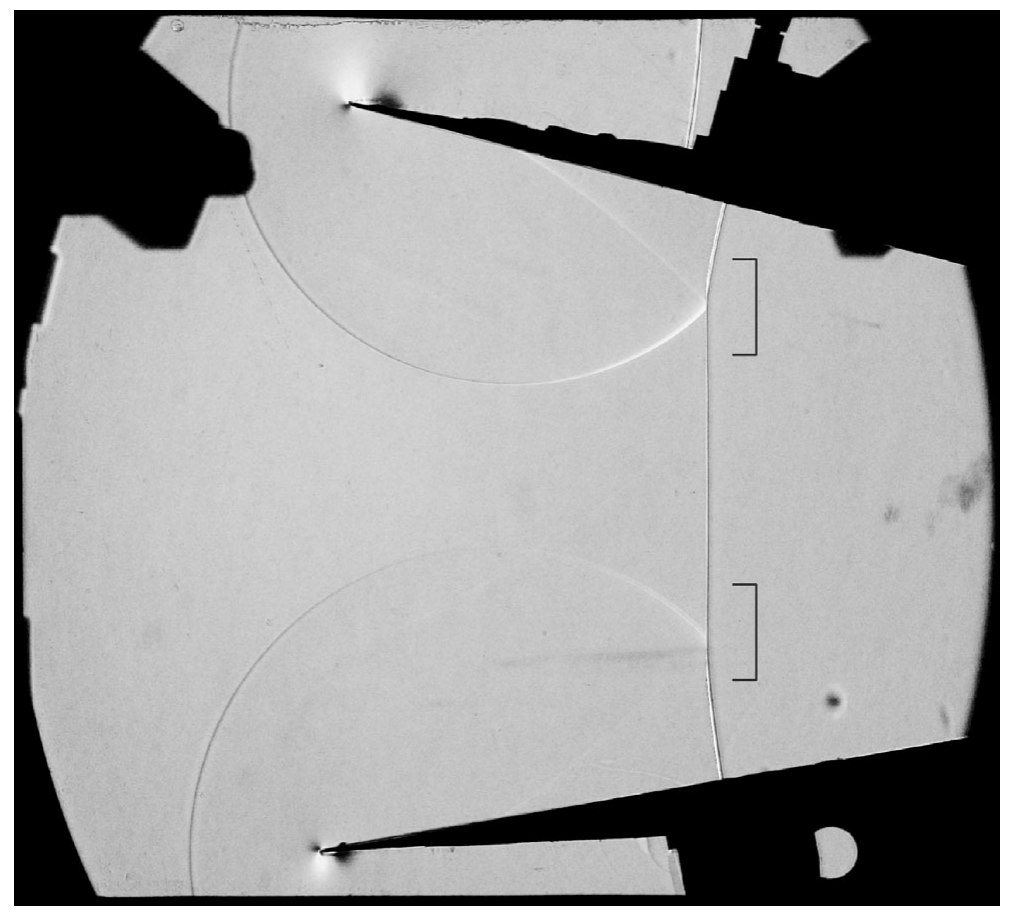

Figure 3. Run $68, M_{i}=1.49$ shock in $\mathrm{N}_{2}$ travelling from left to right. Wedge angles are $9.8^{\circ}$ (lower) and $14.6^{\circ}$ (upper). Brackets mark the right edge of the close-ups in figure 4.

(a) Upper reflection

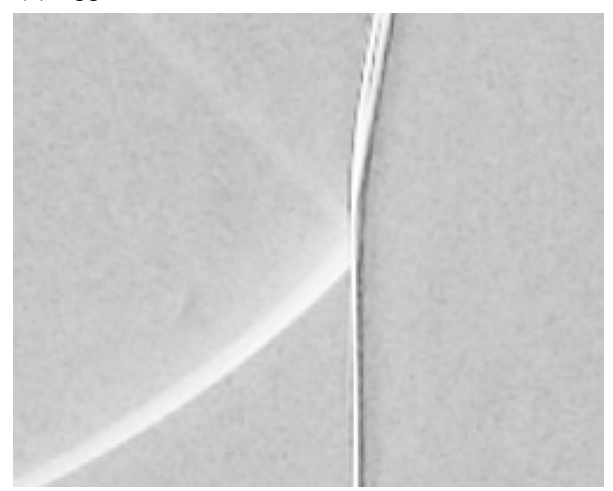

(b) Lower reflection

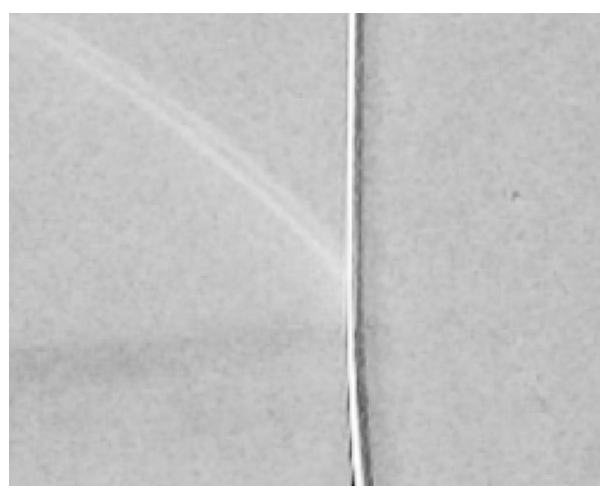

FigURE 4. Run 68, close-ups of the triple-point regions in both reflections.

wedge plates. Also, barely visible are a set of very weak waves that are reflections off some roughness at the test section/cookie cutter interface. The Mach stem just below the leading edge of the reflected wave appears to have finite curvature as well, indicating that the reflection is not a simple Mach reflection, but instead a distributed reflection.

Figure 4 shows magnifications of the two triple-point regions. The flow appears to be a distributed compression, much as described by Colella \& Henderson (1990). The Mach stems are not straight, but rather curve as they approach the incident shock, but do not show a clear kink that would indicate a shock at the leading edge of the 


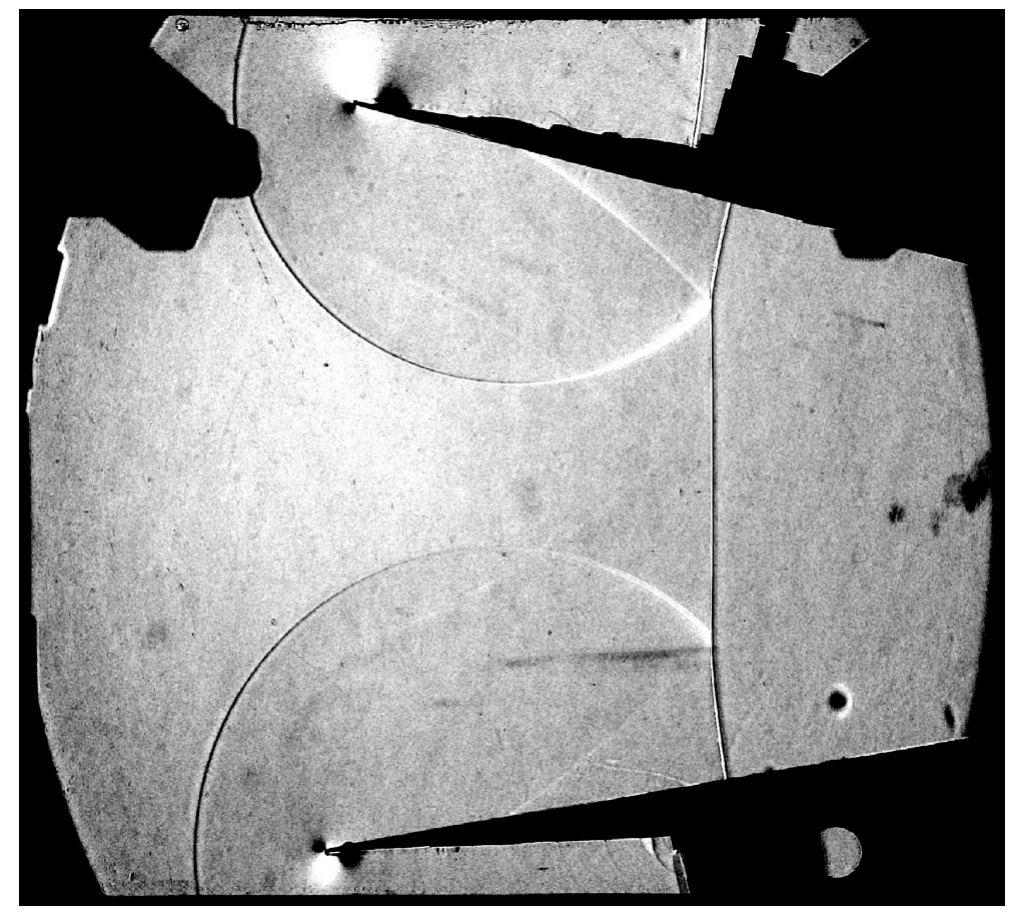

FigURE 5. Run 68, brightened and contrast enhanced to emphasize the sliplines (at the expense of reflected wave details seen in figure 3). Also, more visible are the weak waves originating at the interfaces between the test section and the cookie cutter.

compression. The curvature of the Mach stems does not produce a simple contact surface, but instead a distributed region of non-uniform entropy. The reflection from the upper wedge is stronger with a better-defined leading edge; however, the image cannot distinguish between a steepening compression with or without a very weak leading shock.

Figure 6 shows the incident shock of Run 72, Mach 2.43 in $\mathrm{N}_{2}$ gas. There are notable differences between this and the lower Mach number Run 68. Towards the wedge tips, the bow shocks are nearly attached to the tips. The sliplines are now almost straight, curving back towards the Mach stem, and roll up where they meet the wall. The angle between the reflected waves and the incident shock is notably larger, resulting in a slower relative penetration of the reflected waves into the undisturbed area behind the incident shock. Here the Mach stems appear straight at the scale of the image, producing the appearance of a kink at the triple point.

In the traditional use of three-shock theory in the validation of Mach-reflection behaviour, a local reflected wave geometry such as wave angles and slipline angles is computed from given incident Mach numbers and triple-point trajectories $\left(\chi+\theta_{w}\right.$ in figure 7) and these results are compared with experiments. For the purpose of understanding the structure of the triple point this is excellent, but for looking at the total compression of the reflection this has the distinct disadvantage of ignoring the remaining compression along the Mach stem. Here, to provide a reference that suggests the importance of compression along the Mach stem, rather than fix the triple-point trajectory, the Mach stem is modelled to be straight and perpendicular to the wedge face and the triple-point trajectory is computed using the predictions of three-shock theory. Observed differences from this model are then attributed either to 


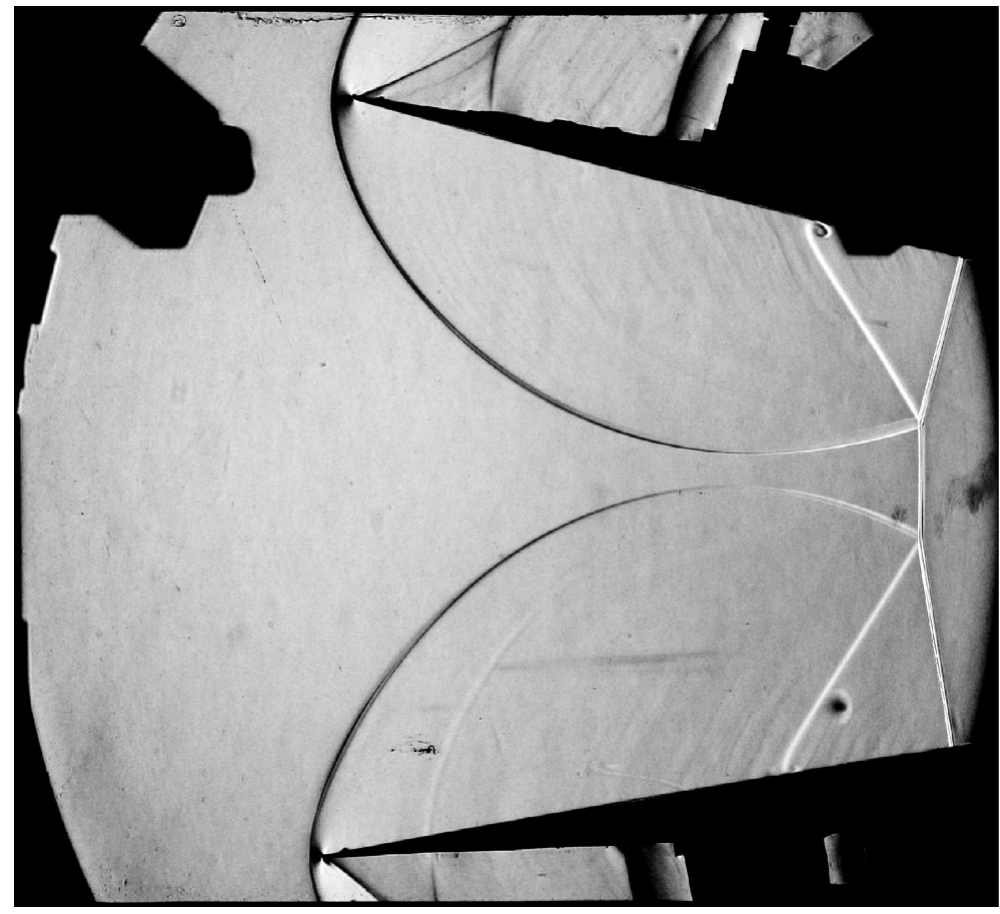

FIGURE 6. Run $72, M_{i}=2.43$ shock in $\mathrm{N}_{2}$ travelling from left to right. Wedge angles are $9.8^{\circ}$ (lower) and $14.7^{\circ}$ (upper). Weak waves appearing to originate from the wedge surface may be due to the limitations and roughness of the gaskets between the wedge and the windows.

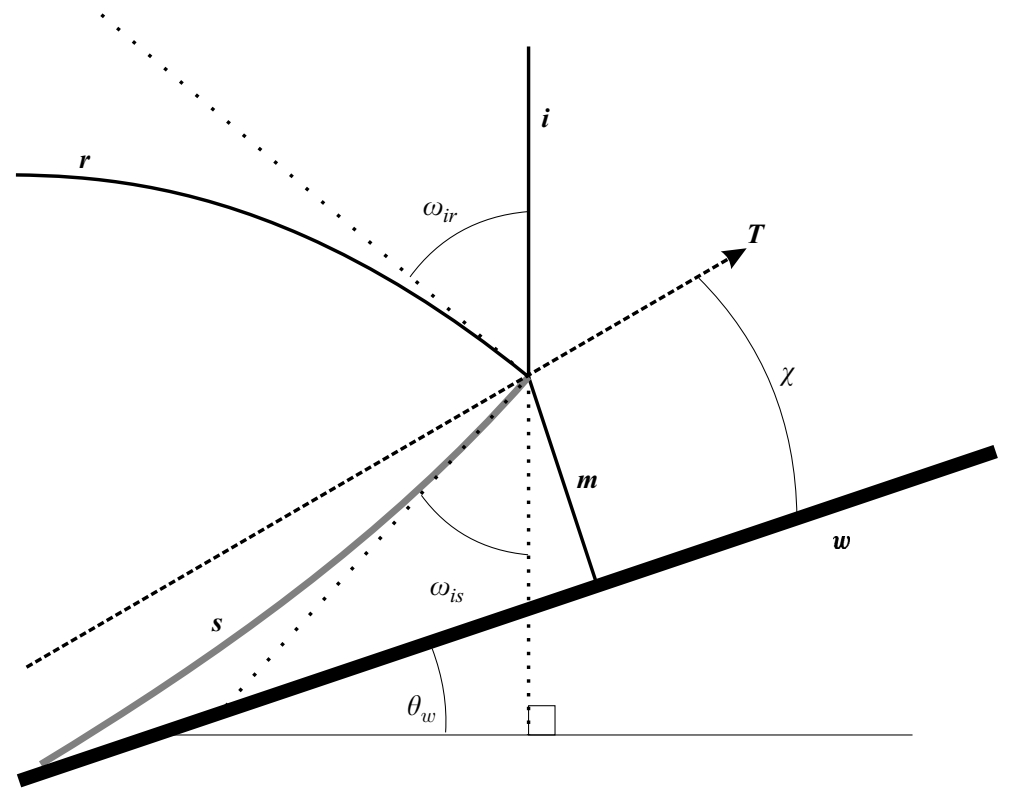

FIGURE 7. The geometry of the triple points. Here, $\boldsymbol{i}$ is the incident shock, $\boldsymbol{r}$ is the reflected shock, $\boldsymbol{m}$ is the Mach stem, $\boldsymbol{s}$ is the slipline, $\boldsymbol{T}$ is the triple-point trajectory and $\boldsymbol{w}$ is the wedge surface. Both $\omega_{i r}$ and $\omega_{i s}$ were measured from the incident shock as its straightness minimized measurement uncertainty. 
the physical curvature of the Mach stem (which indicates a distributed compression), or to the significant internal structure that is not captured by the three-shock model (e.g. a Guderley reflection), or to both. The model indicates what a fully compact reflection would look like, which a highly compact reflection would approximate. At the other extreme, a distributed reflection (where the majority of the compression occurs away from the triple point) would agree quite poorly with the model.

Note that the model system admits two branches in $M_{i}, \theta_{w}$ space: on the one branch, the reflected wave is backward tilted and the trajectory angle increases with increasing $M_{i}$. However, on the other branch, the modelled reflection is forward tilted and the trajectory angle increases with decreasing $M_{i}$. In the context of Mach reflections, the increase of the trajectory angle with decrease in the incident Mach number is unphysical. This suggests that conditions in the parameter range of the second branch produce reflections not captured by the model.

The geometry of the triple point is shown in figure 7, where the measured (also modelled) angles are shown: $\omega_{i r}$, the angle between the incident shock, $\boldsymbol{i}$, and the (tangent to the) reflected wave, $\boldsymbol{r} ; \omega_{i s}$, the angle between the (extension of the) incident shock and the (tangent to the) slipline, $s$; and $\chi$, the angle between the trajectory of the triple point, $\boldsymbol{T}$, and the wedge surface, $\boldsymbol{w}$. Model results are calculated by selecting $\theta_{w}$ and $M_{i}$ and iterating to find a value of $\chi$ that produces a valid solution. These model results do include conditions that may violate the Colella \& Henderson (1990) vNR conditions that require that $\omega_{i s}+\omega_{i r}>90^{\circ}$, which, as noted previously, is an unphysical solution branch.

The incident shock was measured over many experiments at multiple conditions and was consistently found to be at $0.2^{\circ}$ away from vertical, with the shock at the top of the test section slightly leading the shock at the bottom; this was consistent with timing measurements at the inlet of the test section. While the direction of gravity is, of course, irrelevant to the study at hand, an accurate orientation of the coordinate system is important for the comparison of the results of experiment and simulation. The triple-point trajectory is measured for this study as the trajectory of the intersection of the very first part of the reflected wave with the incident shock. Tangents to the reflected wave and the slipline must be constructed, and this is further complicated by the diffuse nature of the slipline. These tangents are again taken from the leading edge of the reflected wave and from the edge of the slipline associated with that leading edge. All angles were measured on the corrected images within the image manipulation program Photoshop CS2. (Details of image processing are given in Appendix B.)

The results for the N1.5 case are shown in table 4 . There are fairly large deviations of the experimental results from the model predictions, but this is to be expected, since the model results in a forward-tilted reflection $\left(\omega_{i s}+\omega_{i r}<90^{\circ}\right)$ that is expected to be unphysical. Furthermore, the experimental image (figure 3) results show distributed reflected waves, which are at odds with the simple three-shock description. Note that $\omega_{s l}+\omega_{i r} \approx 90^{\circ}$, within uncertainty, in the experiment; whether the observed reflection is forward- or backward-tilting is unresolved.

While the images do not give a clear resolution to the question of the absence or presence of a shock leading the distributed reflection, an upper bound on the possible strength of such a shock can be given by the Mach number of the triple point relative to the flow behind the incident shock. In this case, that Mach number is calculated as $\approx 1.025$ for both the upper and lower reflections. However, a simple reflected shock at that Mach number would yield a $2.8^{\circ}$ difference in the incident shock and Mach stem angles at the triple point that is not seen. Either the leading-reflected wave is 


\begin{tabular}{cccccc} 
& \multicolumn{2}{c}{ Lower wedge } & & \multicolumn{2}{c}{ Upper wedge } \\
\cline { 2 - 3 } \cline { 5 - 6 }$\theta_{w}$ & \multicolumn{2}{c}{$9.8 \pm 0.1$} & & \multicolumn{2}{c}{$14.6 \pm 0.1$} \\
\cline { 2 - 3 } \cline { 5 - 6 }$\chi+\theta_{w}$ & $29.8 \pm 0.2$ & 33.5 & & $29.8 \pm 0.2$ & 34.3 \\
$\omega_{i r}$ & $45.1 \pm 0.7$ & - & & $44.6 \pm 0.7$ & - \\
$\omega_{i s}$ & $44.2 \pm 0.7$ & - & & $44.6 \pm 0.7$ & - \\
$M_{t p}$ & $1.025 \pm 0.003$ & - & & $1.025 \pm 0.003$ & -
\end{tabular}

TABLE 4. Measured reflection properties for Run $68, M_{i}=1.49$ shock in $\mathrm{N}_{2}$, compared with a straight Mach stem model using the three-shock theory. The disagreement is expected, as the model has for a forward-tilted reflected shock. $M_{t p}$ is the Mach number of the triple point relative to the flow behind the incident shock. No model results are listed for triple-point structure, as the model is only used to clearly indicate how far the observed flow is from a straight Mach stem flow.

\begin{tabular}{lccccc} 
& \multicolumn{2}{c}{ Lower wedge } & & \multicolumn{2}{c}{ Upper wedge } \\
& \multicolumn{2}{c}{$9.8 \pm 0.1$} & & \multicolumn{2}{c}{$14.7 \pm 0.1$} \\
\cline { 2 - 3 } \cline { 5 - 6 }$\theta_{w}$ & Measured & Model & & Measured & Model \\
$\chi+\theta_{w}$ & $28.3 \pm 0.2$ & 29.1 & & $29.9 \pm 0.2$ & 30.7 \\
$\omega_{i r}$ & $66.2 \pm 0.7$ & 66.4 & & $72.9 \pm 0.7$ & 75.2 \\
$\omega_{i s}$ & $31.9 \pm 0.7$ & 29.5 & & $32.1 \pm 0.7$ & 29.5 \\
$\mathrm{M}_{t p}$ & $1.048 \pm 0.003$ & - & & $1.102 \pm 0.003$ & -
\end{tabular}

TABLE 5. Measured reflection properties for Run $72, M_{i}=2.43$ shock in $\mathrm{N}_{2}$, compared with a straight Mach stem model using the three-shock theory.

significantly weaker than that or there is a more complicated structure at the triple point that significantly reduces the pressure rise; notably, for the measured trajectory angle the triple-point configuration would be, according to Vasilev et al. (2008), a Guderley reflection.

The N2.5 case results are shown in table 5. The agreement with (a straight Mach stem) model is much better, as would be expected from the nearly straight Mach stems. The measured triple-point trajectories, although very low for a fully straight Mach stem, still produce shock polars consistent with a standard Mach reflection. (However, for the lower wedge angle, a forward-tilting solution to the three-shock equations is possible for triple-point trajectories within measurement uncertainty.) The upper limit of the reflected shock Mach numbers is the Mach number of the triple point relative to the flow behind the incident shock; for the lower and upper reflections, those Mach numbers are calculated as $\approx 1.05$ and $\approx 1.10$, respectively.

\subsection{Pressure traces}

A typical pressure trace from the N1.5 case is shown in figure $8(a)$. The transducer (L4) is located on the lower plate at a distance of $7.6 \mathrm{~cm}$ from the apex of the wedge. The major features are annotated and they consist of the pressure rise from the incident wave structure (initially a Mach stem) followed by the three separate reflections of the reflected waves, and finally by the returning shock. The width of the pressure rise of the incident shock is a function of the finite size of the pressure-sensing element and the time required by the shock to cross the element.

Since pressure-rise profiles depend both upon the geometry of the wave/transducer interaction and the internal pressure profile of the wave, some understanding of the internal structures of the reflected waves and how they give rise to the pressure 
(a) Run $68, M_{i}=1.49$

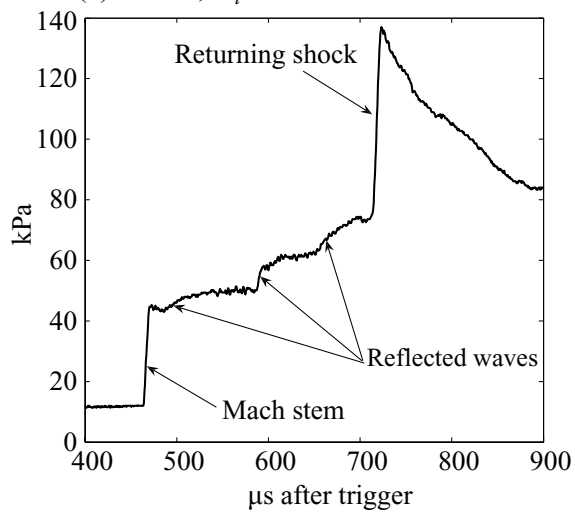

(b) Run $72, M_{i}=2.43$

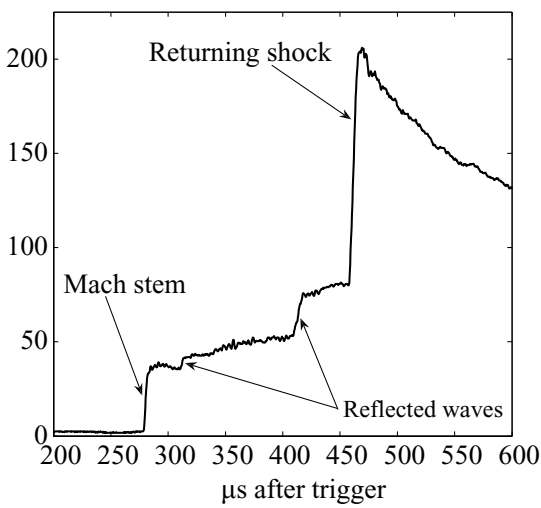

FIGURE 8. Annotated pressure traces from transducer $L 4$ located $7.6 \mathrm{~cm}$ from the apex.

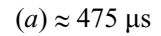

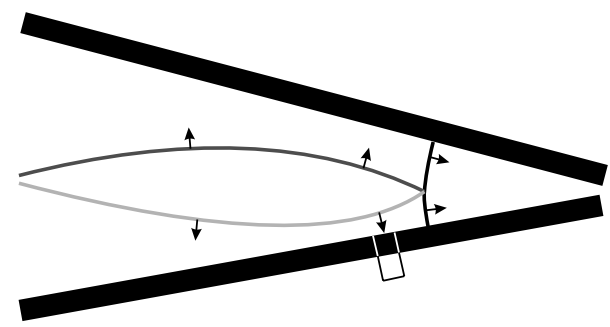

$(a) \approx 650 \mu \mathrm{s}$

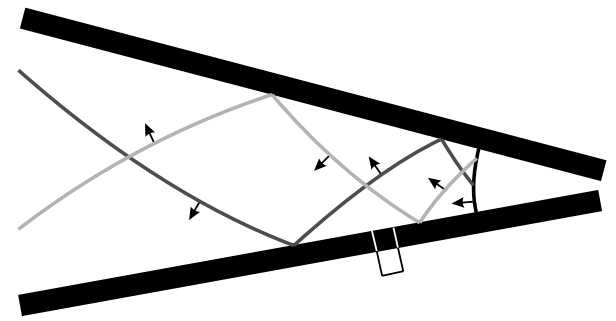

FigURE 9. Sketches of wave structures (abstracted from the computation of Run 68) relevant to the typical pressure traces. The location and relative width of the L4 transducer is indicated by a small rectangle, the dark grey line is the wave initially reflected from the upper $\left(14.8^{\circ}\right)$ wedge and the light grey line corresponds to the wave initially reflected wave from the lower $\left(9.8^{\circ}\right)$ wedge. Arrows show the direction of wave movement. Triple points cross (for the second time) in $(a)$ and the returning shock can be seen as the rightmost feature in $(b)$.

trace seen in figure 8 may be achieved simply by abstracting the leading edges of the reflections from computations (these computations are described in detail in $\S 5$ ). figure $9(a)$ shows the flow at a time $\sim 475 \mu$ s after the trigger; after the initial Mach stem has crossed transducer L4, but before the first reflected wave. The pressure rise seen in the trace at $\sim 500 \mu$ s is due to the reflected wave initially associated with the lower wedge. Note that this wave impacts the transducer at a nearly normal incidence; thus the gradual form of the pressure rise is not because of the transducer's finite size. Figure $9(b)$ shows the reflections $175 \mu$ s later, just after the second reflected wave hits the transducer. In this figure, the stronger wave, initially reflected from the upper wedge, has just passed the transducer and will be followed by the lower wedge reflection and the returning shock. The difference in pressure profiles is not attributable to the geometry of the wave/transducer interaction, but is instead indicative of a structural difference between the waves reflected off the upper and lower wedge walls.

A typical pressure trace for a N2.5 case (Run 72) is shown in figure $8(b)$. Again, the general pattern of Mach-stem/reflected-waves/primary-shock-return is found on this trace. As a result of the lower penetration of the reflected waves into the area behind the incident shock, only two reflected waves are visible on the trace. 


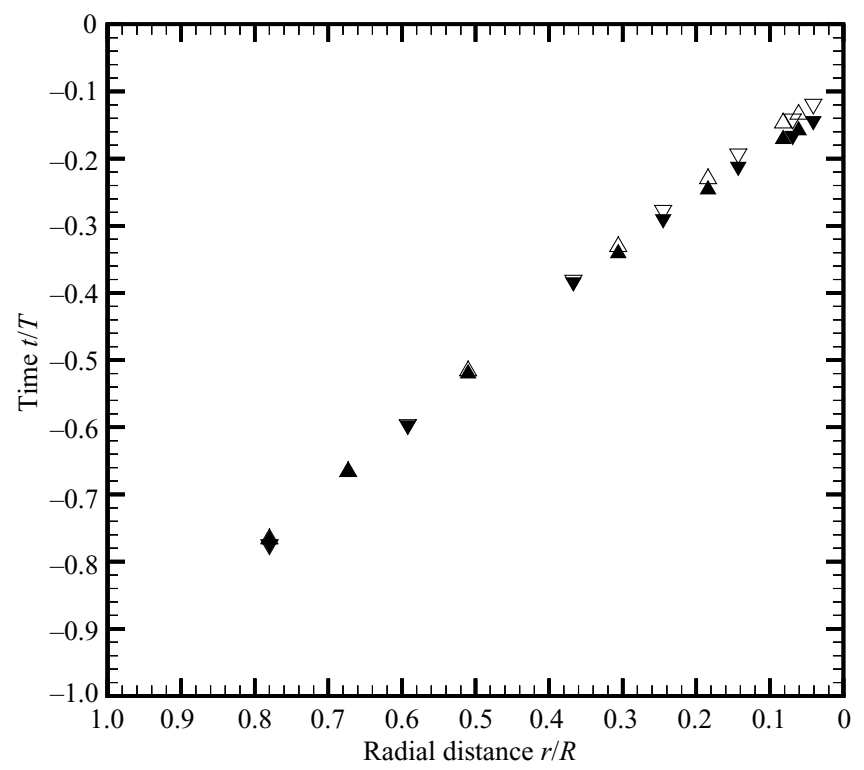

FIGURE 10. Cross condition timing comparison: N1.5 (Run 69, open symbols) vs. N2.5 (Run 71, closed symbols). Triangles correspond to upper plate data and inverted triangles correspond to lower plate data. Only by the sixth transducer is there any visible distinction.

The most precise measurements that the pressure transducers provide are the arrival times of the Mach stem of the incident shock and the return of the incident shock. The typical experimental uncertainty for the arrival times is $\approx 1-2 \mu \mathrm{s}$. The arrival times can be used to create an $(r, t)$ diagram of the leading shock position as the flow evolves. Figure 10 shows the incoming normalized shock arrival times for two different runs. Note that the Mach numbers of the incident shocks for the flows shown here are $M_{i}=1.48$ and $M_{i}=2.46$, yet the collapse of the incoming shock arrival times is fairly good. The N2.5 case does accelerate slightly faster, but the similarity is striking. Time and distance are normalized by the shock speed and the wedge dimensions in such a way that, if there were no wedge, the incident shock would reach the location of the wedge axis at (normalized) $t=0$, following a straight line from the lower left corner of the figure to the upper right corner. In this way, the acceleration that results from shock focusing can be readily seen. We shall return to shock arrival times as a metric for shock focusing in $\S 6$.

\section{Computational methodology}

The numerical simulations utilize a dynamically adaptive second-order Eulerian Cartesian finite-volume upwind scheme that allows for geometrically complex boundaries using a volume-of-fluid approach. The boundary geometry is exposed onto a Cartesian mesh by employing a scalar level-set function that stores the signed distance to the boundary surface. Multiple distinct numerical integration schemes for the Euler equations have been incorporated into a parallel block structured meshrefinement algorithm that provides very high local spatial and temporal resolutions at runtime. The overall method is tailored for high-resolution simulation of compressibleflow phenomena in evolving geometries undergoing arbitrary topology changes (Deiterding et al. 2006). 


\subsection{Numerical method}

For the purpose of simulation, the two-dimensional compressible Euler equations are integrated numerically. This excludes the effects of viscosity (e.g. boundary layers on the wedge plates and viewing windows). Written in a conservative form, the two-dimensional Euler equations are

$$
\frac{\partial \boldsymbol{q}}{\partial t}+\frac{\partial \boldsymbol{F}}{\partial x}+\frac{\partial \boldsymbol{G}}{\partial y}=0
$$

in which $\boldsymbol{q}=(\rho, \rho u, \rho v, E)^{\mathrm{T}}$ and the flux vectors are given by

$$
\boldsymbol{F}=\left(\begin{array}{c}
\rho u \\
\rho u^{2}+p \\
\rho u v \\
u(E+p)
\end{array}\right), \quad \boldsymbol{G}=\left(\begin{array}{c}
\rho v \\
\rho u v \\
\rho v^{2}+p \\
v(E+p)
\end{array}\right)
$$

where $\rho$ is the density, $p$ is the pressure and $u, v$ are the velocities in the $x$ and $y$ directions, respectively. The total energy $E$ is related to internal energy per unit mass, $e$, and the velocities by

$$
E=\rho e+\frac{1}{2} \rho\left(u^{2}+v^{2}\right) .
$$

For all the simulations of shocks in nitrogen reported, the ideal equation of state, $p=\rho R T$, is assumed and the internal energy is given by $e=c_{v} T$. The gas properties used for $\mathrm{N}_{2}$ are summarized as follows: $R=296.8 \mathrm{~J}(\mathrm{~kg} \mathrm{~K})^{-1}, c_{v}=c_{p}-R$ and $\gamma \equiv$ $c_{p} / c_{v}=1.4$.

A hybrid scheme is used to represent the spatial derivatives of the flux vectors $\boldsymbol{F}$ and $\boldsymbol{G}$. In this approach, as with all finite-volume methods, a derivative is computed as the difference of interpolated flux values on 'cell walls' located half-way between grid points:

$$
\frac{\mathrm{d} f}{\mathrm{~d} x}=\frac{1}{\triangle x}\left(f_{j+1 / 2}-f_{j-1 / 2}\right) .
$$

As described by Pantano et al. (2007), the scheme is a high-resolution approach specially constructed to have a very low numerical dissipation. It computes these interpolated values either by a WENO method at the shocks or by a centre-difference method consistent with a skew-symmetric formulation in smooth regions of the flow. The time integration is performed by the use of a third-order strong stability preserving (SSP) Runge-Kutta scheme (Gottlieb, Shu \& Tadmor 2001). For additional verification, a simple HLL finite-volume scheme incorporated in the same adaptive framework was also used. In all verification cases, the results were found to be in good agreement.

Mathematically, the boundary condition for the Euler equations at rigid boundaries is simply $\boldsymbol{n} \cdot \boldsymbol{u}=0$, where $\boldsymbol{n}$ is the normal vector at the boundary. Numerically, this is enforced by an implementation of the 'Ghost Fluid Method'. Mirroring of the flow is applied between time steps, which allows the fluid solver to integrate each time step over the entire domain (fluid region and ghost-fluid region). This approach has the advantage that the boundary conditions are applied in a manner that is separated from the actual mechanics of the solver in question and can be applied to complicated evolving geometries. While the normal-velocity condition is only satisfied to first order, the simulation produces excellent agreement with experiment, both qualitatively and quantitatively. 
(a) Pressure contours for lower reflection

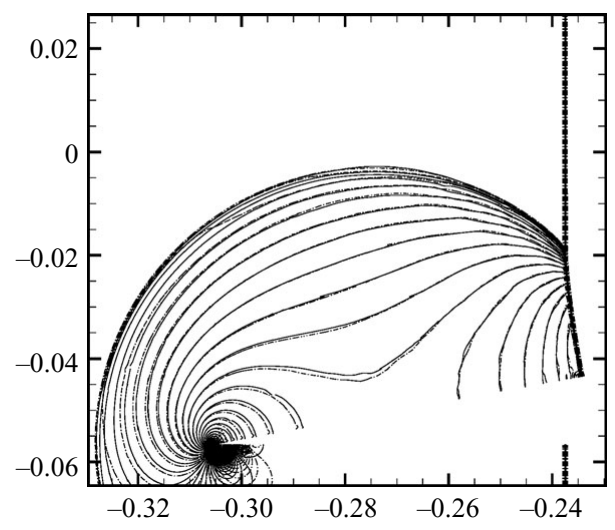

(b) Pressure at L5 transducer location

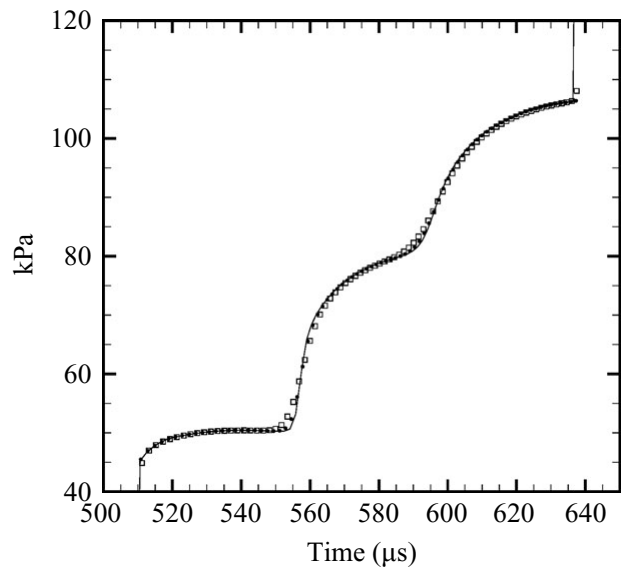

FIGURE 11. Self-convergence of the pressure field. In $(a)$ resolutions of $\Delta x=0.03125 \mathrm{~mm}$ (solid line) and $\triangle x=0.125 \mathrm{~mm}$ (dash-dotted line) are shown, while in $(b)$ resolutions of $\triangle x=0.03125 \mathrm{~mm}$ (solid line), $\triangle x=0.0625 \mathrm{~mm}$ (squares) and $\triangle x=0.125 \mathrm{~mm}$ (circles) are shown.

\subsection{Simulation methodology and comparison with experiment}

To allow for a comparison with experiments, the experimental diagnostics were modelled within the numerical framework. Synthetic transducers recorded the pressure history at locations corresponding to the centres of the physical transducers, with a sampling rate of approximately $1 \mathrm{MHz}$. Additionally, the full state vector is recorded at predetermined times that correspond to the schlieren images; from the instantaneous density field, artificial schlieren images are calculated. The additional computed quantities, such as the velocity and pressure fields, allow for a more comprehensive investigation of the shock physics than provided by experiment alone.

Although the Euler equations are scale invariant, the simulations were performed directly in MKS units for simplicity of set-up and ease of interpretation and comparison with experiment.

Initial conditions were defined by the state of the undisturbed gas and the measured speed of the shock at the timing transducer $33.02 \mathrm{~cm}$ (13 in) from the wedge apex. This information is summarized in table 3 . The boundary conditions comprise the measured wedge angles in the experiment combined with a zero-gradient condition on the open end of the computational domain. We note that the interior of the computational wedge matches the experiment down to the 0.25 in hinge radius but no attempt was made to match the exterior wedge with the complicated geometry and support structures in the experiment as these features are essentially irrelevant to the flow in the wedge interior during the experiment.

While there is an inherent experimental uncertainty in the measured conditions such as wedge angle and Mach number, no attempt was made to exploit this in maximizing agreement between experiment and simulation. For the sake of brevity, only some representative comparisons between the simulation and experiment are presented.

To ensure that the flow features were well captured, the simulations were performed at several resolutions and self-convergence of the solutions was monitored. Figure 11 $(a)$ shows the pressure contours of the lower Mach reflection in Run 68 computed at two different resolutions and presented at $t=248.4 \mu$ s to correspond with the schlieren figure 3. As can be seen, results at the two resolutions are in good 
(a) Shock arrival times

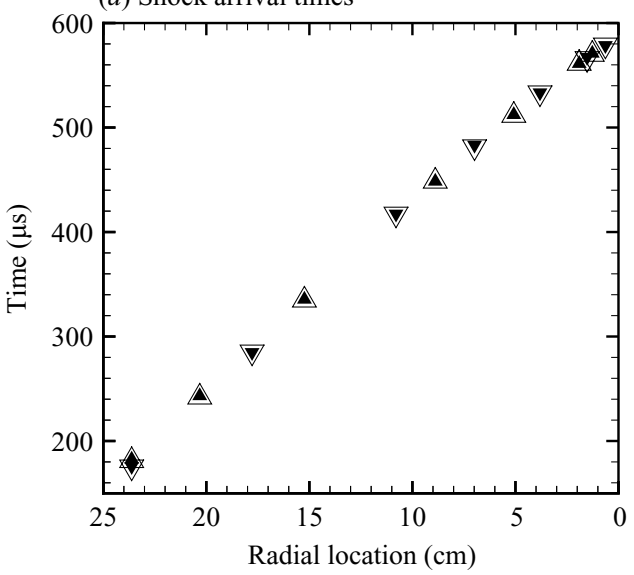

(b) Close-up with error bars

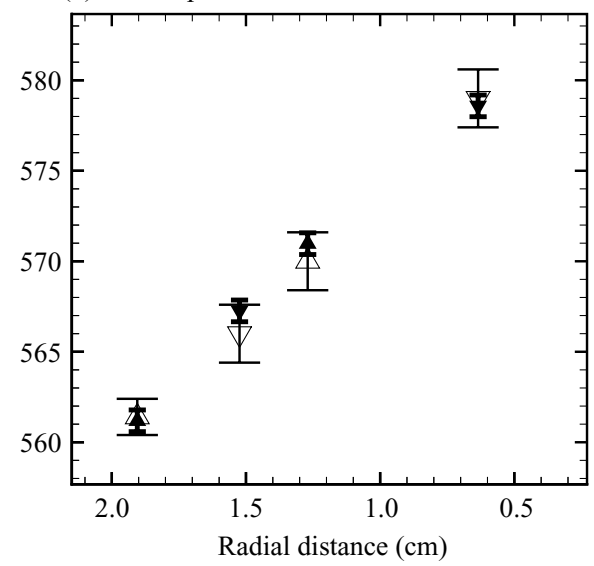

FIGURE 12. Incoming shock location vs. time for a $M_{i}=1.43$ shock travelling in $\mathrm{N}_{2}$. Open symbols represent experimental data and solid symbols correspond to simulation data. The error bars with wide crosses denote the experimental uncertainty and the narrow error bars result from the computational sampling frequency.

agreement. Examining the flow history much closer to the apex of the wedge provides a more demanding metric of convergence. Here, the effective resolution, defined as the number of grid points spanning the wedge, is significantly reduced. Figure 11(b) presents the pressure history as recorded from simulations at three different resolutions at a point that corresponds to the centre of the L 5 transducer, approximately $1 / 7$ th of the total wedge distance from the apex. Here, one can see more clearly the first-order convergence typical of all shock capturing methods in the region of shocks. We found the resolution of $\triangle x=0.0625 \mathrm{~mm}$ to be acceptable and it was used for comparison with the experiments. Such a spacing places approximately 40 grid points across the exposed $25^{\circ}$ of the hinge at the apex. Results at this resolution were not shown in figure 11(a) as they are virtually indistinguishable from those at the higher resolution.

As stated earlier, the most precise experimental measurements are the times at which the Mach stem crosses each transducer. Figure 12 compares simulation and experiment for these arrival times. In all cases, the data are within both measured and computed uncertainty. We also examine the full pressure history as recorded by the transducers located in the inner half of the wedge. In the experiments, these transducers were found to be the least sensitive to the solid vibration of the wedge itself. An examination of figure 13 shows agreement between the transducer data and the simulation. The agreement is particularly good prior to the arrival of the returning shock. We note that after the focusing experiment is completed and the main shock returns outward, the pressure spikes that indicate the passage of the returning shock consistently occur sooner in the simulation. A brief description of the returning shock and complications in comparison are presented in Appendix A.

\section{Interpretation and discussion}

The combined experimental and computational results will be discussed in three parts: first, the initial phase that produces two independent pseudo-steady Mach reflections; second, the persistent effects of the initial reflected wave structure on the subsequent re-reflections off the wedge; and finally, the overall character of the shock-wave focusing generated by this process. 
(a) Upper transducer, U5

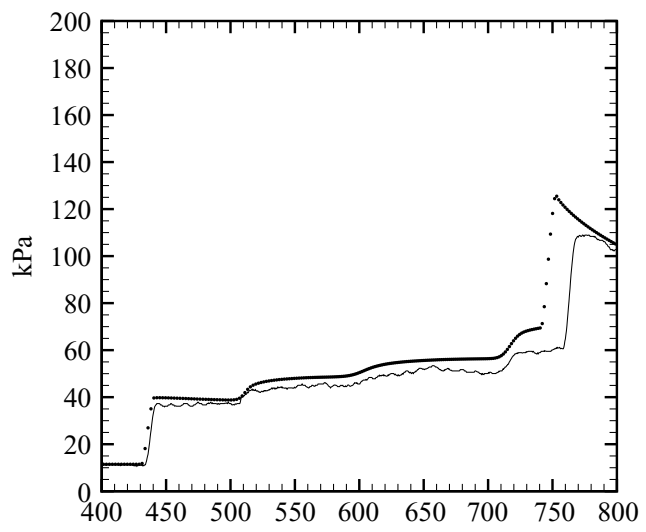

(c) Lower transducer, L5

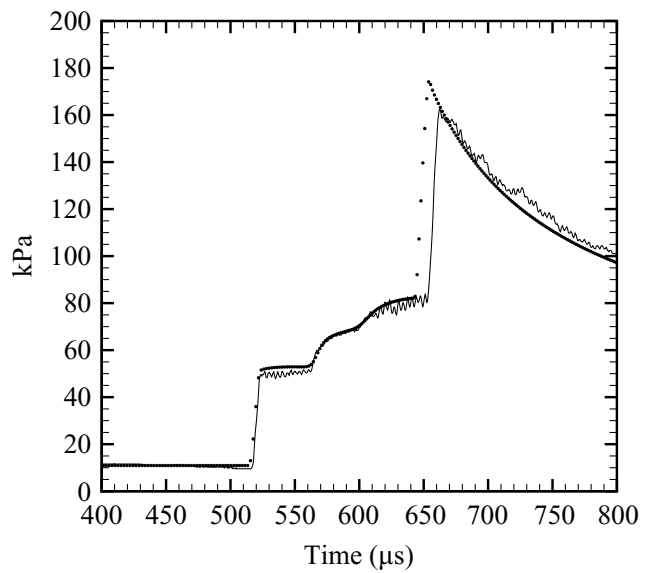

(b) Upper transducer, U6

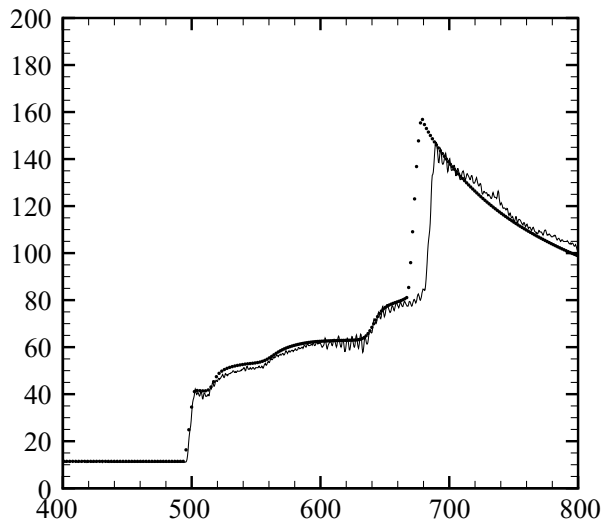

(d) Lower transducer, L6

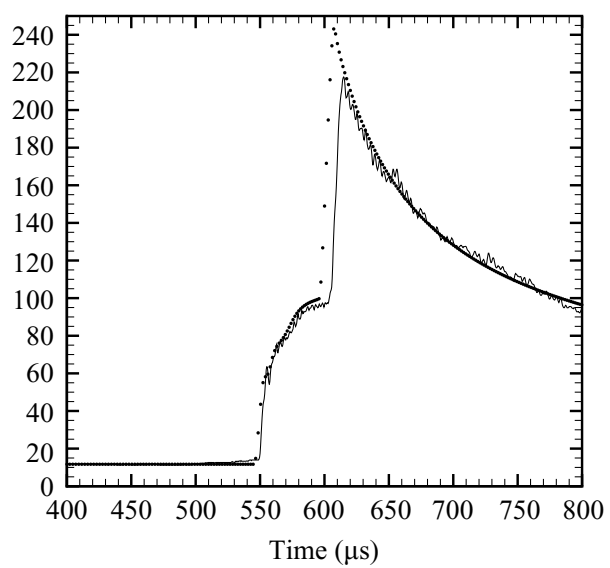

FIGURE 13. $M_{i}=1.48$ shock in $\mathrm{N}_{2}$ : A comparison of the simulated pressure traces (circles) with the experiment (lines) for the fifth and sixth transducers on the top and bottom plates. The large spike with gradual decay corresponds to the outgoing passage of the primary shock after reflection from the wedge apex.

\subsection{Initial reflection of incident shock}

As discussed in $\S 4$, the initial phase of the shock focusing is a pair of pseudo-steady Mach reflections. While the reflected waves in experiments N1.5 and N2.5 appear to be similar, they are characterized by significant differences as to be expected from the difference in the incident Mach number. These waves range from a weak reflection with a substantial distributed compression wave (the N1.5 case on the $10^{\circ}$ wedge) to shocks with some small distributed waves following (the N2.5 case, both wedges). The structure of these waves will continue to be important in the discussions of the later stages of the flow. Additional differences can be seen in the shape and form of the slipline, as mentioned earlier. The simulation data aid in understanding of these features.

Figures $14(a)$ and $14(b)$ reproduce the schlieren images in figures 3 and 6, with overlaid pressure contours from the numerical simulations. Pressure is, of course, continuous across a contact surface (slipline). The small local numerical errors that 
(a) Run $68, M_{i}=1.49$

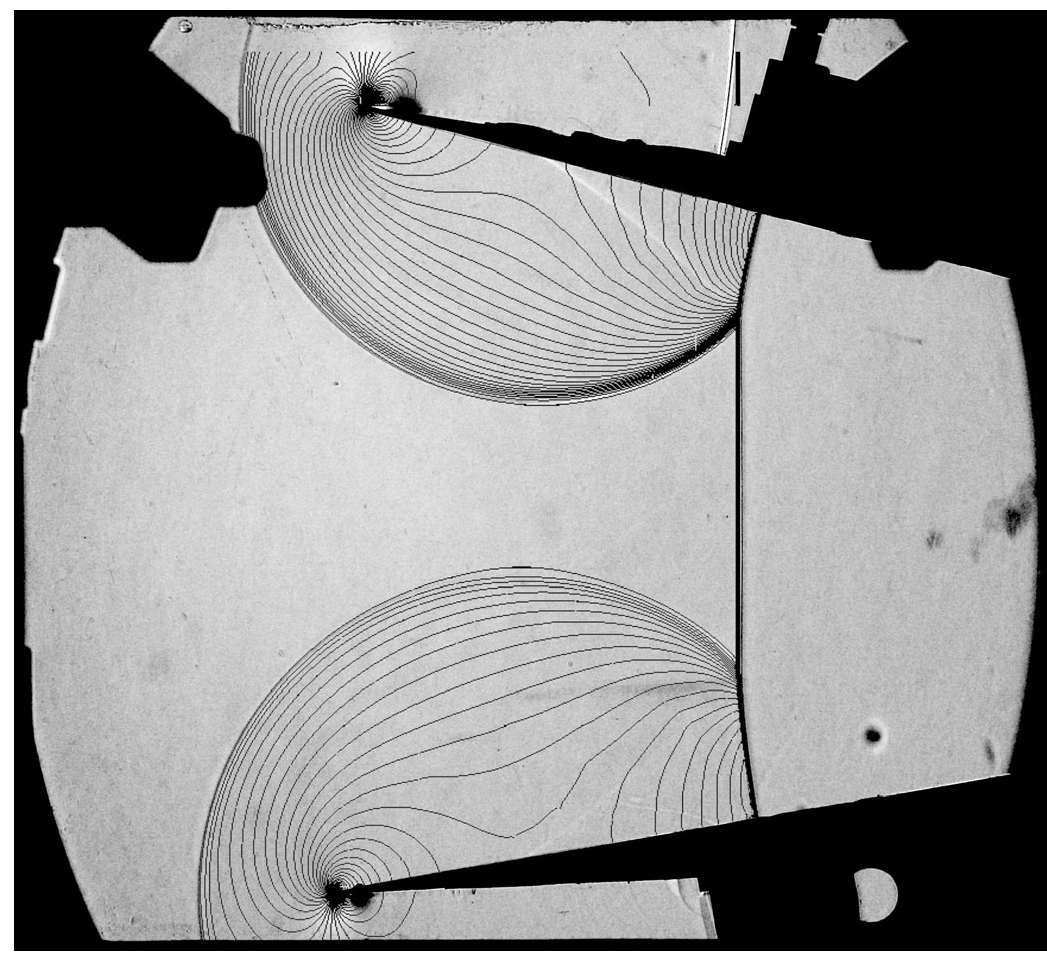

(a) Run $72, M_{i}=2.43$

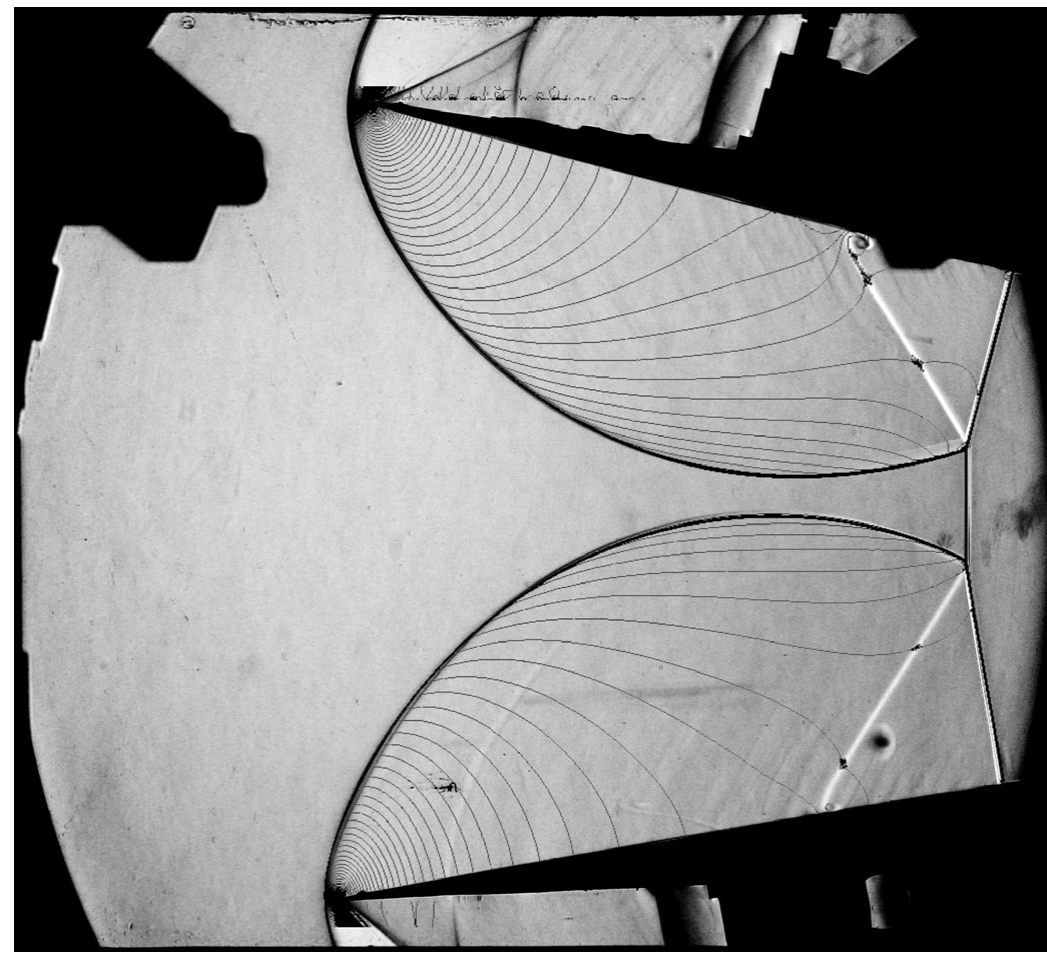

FigURE 14. Schlieren data overlaid with simulated pressure contours. 
(a) Experiment

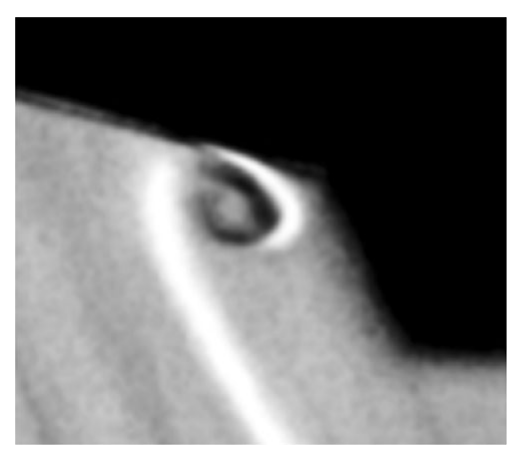

(b) Simulation

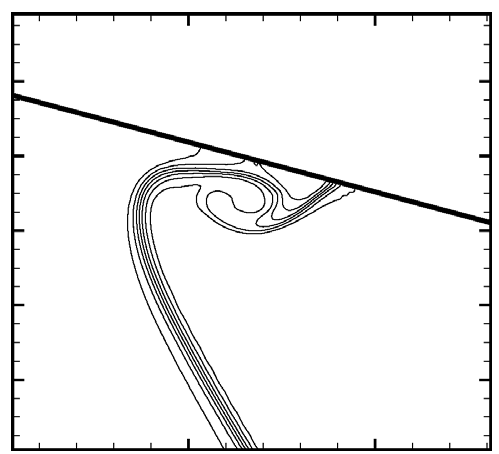

FIGURE 15. Comparison of the slipline roll up in Run 72 shows minor differences due to viscous effects. Density contours are shown in the image from the simulation. The apparent object in the schlieren, to the right of the roll up, is the shadow of a mirror clip.

are inconsequential in the estimation of the pressure field serendipitously mark the contact surface, permitting a precise comparison of its location between experiment and simulation (cf. figure 14b). The contours for Run 68 (Mach 1.49) show that there are two competing high-pressure regions: the first at the tip of the wedge, which turns the upstream flow to accommodate the wedge, and the second that accelerates the downstream flow at the base of the Mach stem. As neither dominates, the result is a pressure along the wall that falls after the initial jump as the Mach stem passes and continues to decrease until after the slipline passes. As the flow in this region is accelerating away from the Mach stem, the slipline is curved towards the wedge tips. This curvature opposes the tendency of the vorticity in the slipline to roll up.

In contrast, the Run $72\left(M_{i}=2.43\right)$ overlay shows a different outcome. At the higher incident Mach shock number, the turning flow dominates the Mach stem over much of the flow. The region of decreasing pressure behind the Mach stem does not extend to where the slipline reaches the wall. The slipline is accelerated towards the Mach stem as it approaches the wall, helping the vorticity in the slipline roll up. Limitations in the simulation can be seen in the actual shape of the roll-up as shown in figure 15 . The simulations assume an inviscid flow and use the equivalent of free-slip boundary conditions, whereas in the physical experiment viscosity and the attendant boundary layer and the no-slip condition cause the front of roll-up structure to separate from the wedge plate and produce a more compact spiral. The lifting of the vortex away from the wall (and movement back towards the slipline) has some similarity to the behaviour of the wall jet with increasing Reynolds number in the Navier-Stokes simulations of Vasilev et al. (2004), although there are substantial differences in Mach numbers, Reynolds numbers and wedge angles.

\subsection{Full shock evolution}

Notable in both experimental and computational results is that the different nature of the reflections off the $10^{\circ}$ and $15^{\circ}$ wedge plates is evident in the pressure traces. At $M_{i}=1.5$, the reflected wave initially associated with the lower wedge plate has a more smoothly distributed form at early times, as can be seen in figure 14(a). But an examination of the experimental and numerical simulation pressure traces indicates that this persists well into the wedge. As an example, the reflected waves are visible in both the upper and lower traces of figure 13 as the pressure increases, with the pressure jumps that are alternately somewhat sharp and somewhat smoother. This is 
also seen in the simulations, perhaps most clearly in figure $16(b)$, where the incoming shock has just passed transducer L5. Here, the difference between the two reflected shocks is notable; the pressure gradients associated with the upper wedge wave (now reflecting off the upper wedge) are significantly greater than those associated with the lower wedge generated wave.

Computational images from a simulation corresponding to Run 69 (a N1.5 case) are shown in figure 16. These images are colour-coded by density and have pressure contours as black lines. The first image shows the flow as the Mach stem passes the $\mathrm{U} 2$ transducer. This is shortly after the reflected waves have first interacted, but before the triple points meet. Notable is the difference in the strength of the two shocks, and that the reflection from the upper wedge is much more clearly defined than the lower wedge reflection. As the flow progresses into the wedge, the high-pressure region where the reflections overlap will reach the incident shock front and strengthen it.

The second image in figure 16 shows the flow as the shocks reach L5, nearly $300 \mu$ s later. In this case, the reflection that was originally formed by the upper wedge has just reflected from the upper wedge again, after reflecting from the lower wedge in the intervening time. The triple-point region, however, is still approaching the upper wedge, and the reflection had just recently passed transducer U5. Figure 17 shows the pressure traces from selected wedge transducers; notably, the U5 transducer shows signs of the reflection slightly before L5 sees the incoming shock.

The third image in figure 16 shows the flow as the incident shock hits transducer L6, $32 \mu$ s after the previous image. The two reflected waves are about to hit L5 and U5 transducers, and the steepness of the two waves is significantly different, with the wave originally from the lower wedge significantly distributed. An examination of the L5 and U5 traces in figure 17(a) reveals evidence of the reflections on the L5 and U5 pressure traces near the time of the incident shock at L6. Notably, however, the U5 transducer, which registers the reflection of the lower wedge originated wave, has a smaller and much smoother pressure jump. In contrast, L5 shows a fairly steep initial jump, with some smoother following pressure rise.

Figure 18 shows density fields and pressure contours for three times during the computation corresponding to Run 71 (a N2.5 case). While both reflected waves are sharper than those in the N1.5 case, the reflection originally from the lower wedge is less clearly defined. In the first of these images, the incoming shock reaches transducer L4. At this point, the reflected waves have bounced off the opposite plates but have not yet met again. The central shock has been once strengthened by the crossing of the reflected waves.

The second image in figure 18 is $28 \mu$ s later, as the first shock passes transducer L5. The triple points have crossed since the last image and the central shock has been strengthened a second time. Note that the reflected wave originally from the lower wedge is approaching L4; on the right in figure 17 some of the experimental pressure measurements from Run 71 are shown. Shortly after the shock hits transducer L5, the L4 trace shows a weak and smooth pressure increase, corresponding to this wave reflection.

The final image in this series is recorded $25 \mu$ s later, as the incident wave hits transducer L7. Note the reflecting wave approaching the U4 transducer originated from the upper plate. Again, the pressure traces in figure 17 show transducer U4 registering a reflection shortly after the incident wave hits transducer L7. This pressure jump is stronger than the lower wedge reflection referred to in the preceding paragraph. 
(a) $t=237 \mu \mathrm{s}$

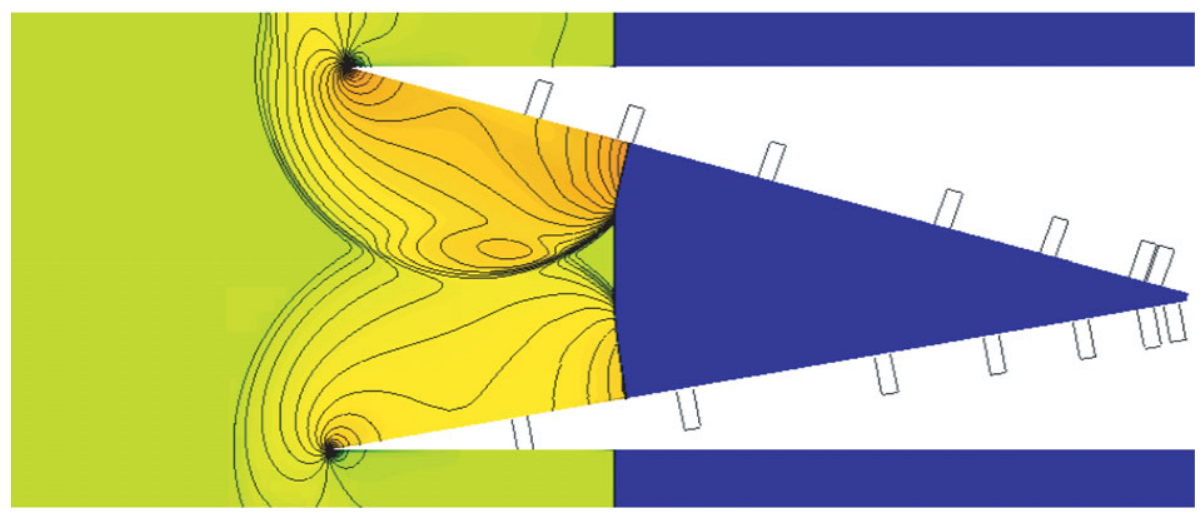

(b) $t=520 \mu \mathrm{s}$

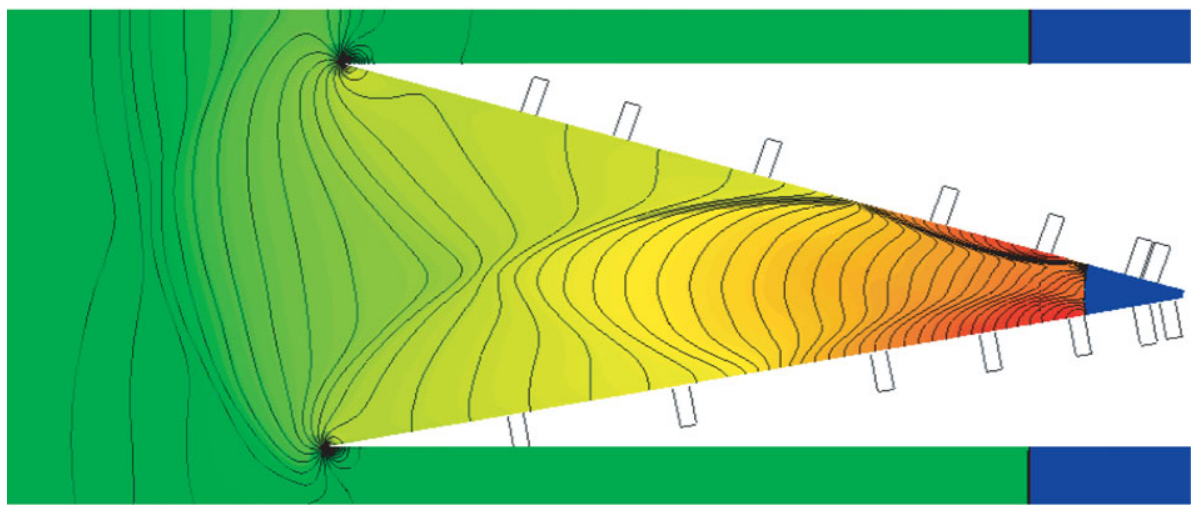

(c) $t=552 \mu \mathrm{s}$

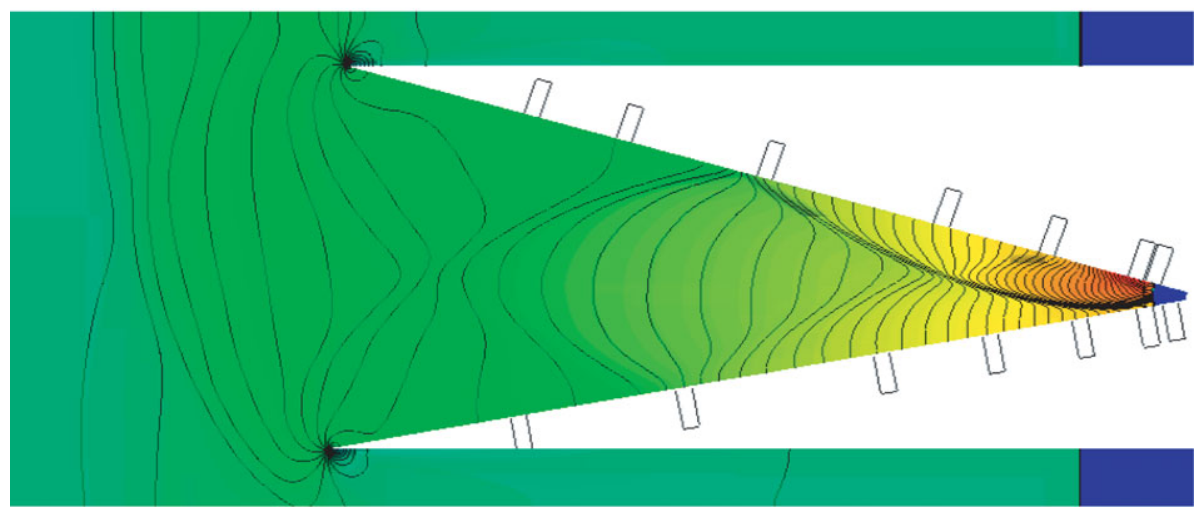

FIGURE 16. Numerical visualizations of Run $69, M_{i}=1.48$, as incident shocks hit transducers $\mathrm{U} 2(t=237 \mu \mathrm{s}), \mathrm{L} 5(t=520 \mu \mathrm{s})$ and L6 $(t=552 \mu \mathrm{s})$.

\subsection{Shock focusing}

Figure 19 illustrates the strengthening processes responsible for shock focusing: the reflected waves meet and cross in $(a)$, producing a region of increased pressure that is higher than that behind the incident shock. As the triple points approach each other, 
(a) Run $69, M_{i}=1.49$

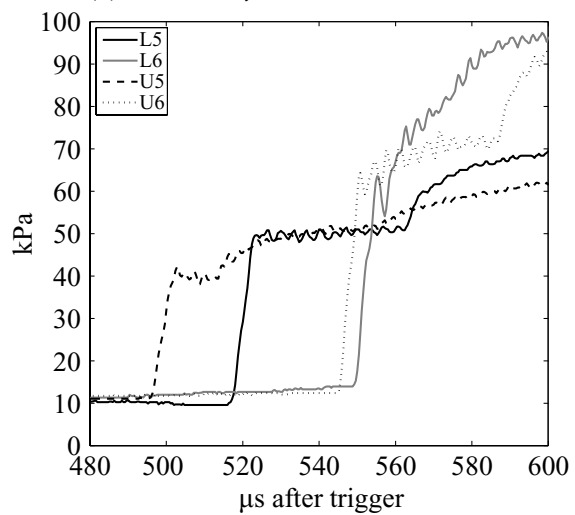

(b) Run $71, M_{i}=2.43$

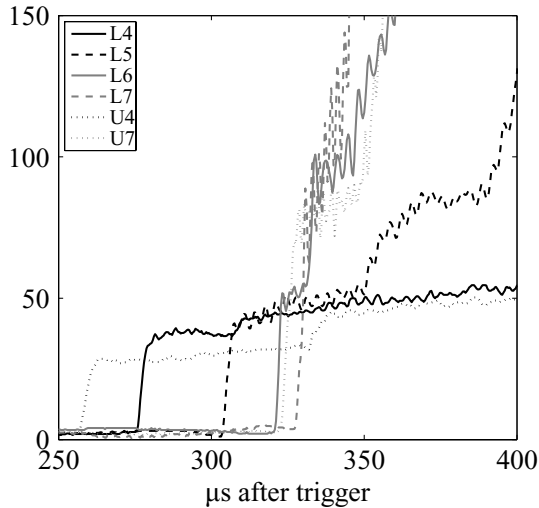

FiguRE 17. Pressure traces corresponding to the times in figures 16 and 18. On the left, upper wall transducer traces are dashed and lower wall transducers are solid. On the right, upper wall transducers are dotted and lower wall transducers are solid or dashed. Oscillations in the pressure traces are seen at the resonant frequency of the physical transducers. Pre-shock signals are caused by structure-transducer coupling.

this region increases in size and approaches the incident shock. After the triple points cross, the configuration appears as shown in figure 19(b), with a new, stronger shock in place of the original incident shock. This process repeats as the triple points (and associated reflected shocks) bounce from the wedge plates and again approach one another to again interact.

This description is simplified by idealizing the reflected waves as simple shocks separating uniform states. However, in the N1.5 case, these waves are not compact and take the form of a distributed compression wave (possibly led by a weak shock). A comparison of figure 19(a) with the first image of figure 16(a) is illustrative. While the general geometry is the same, the actual flow has a more smoothly varying pressure field. Specifically, while the idealized flow has shock strengths that are stepwise varying, the actual flow, with distributed reflected waves, will have a shock strength that increases more smoothly.

When considering shock focusing, it is natural to compare the observed shock positions with the position of both a cylindrical converging shock (as an idealfocusing case) and a planar shock without reflections (as a non-focusing case).

An accurate measure of the Mach history for converging cylindrical shock is given by the shock dynamics theory (Whitham 1957). Here the area $A$ of the shock is related to the Mach number $M$ by,

$$
\frac{A}{A_{0}}=\frac{f(M)}{f\left(M_{0}\right)}, \quad f(M)=\exp \left(-\int \frac{M \lambda(M)}{M^{2}-1} \mathrm{~d} M\right),
$$

where,

$$
\begin{gathered}
\lambda(M)=\left(1+\frac{2}{\gamma+1} \frac{1-\mu^{2}}{\mu}\right)\left(1+2 \mu+\frac{1}{M^{2}}\right), \\
\mu^{2}=\frac{(\gamma-1) M^{2}+2}{2 \gamma M^{2}-(\gamma-1)}
\end{gathered}
$$


(a) $t=273 \mu \mathrm{s}$

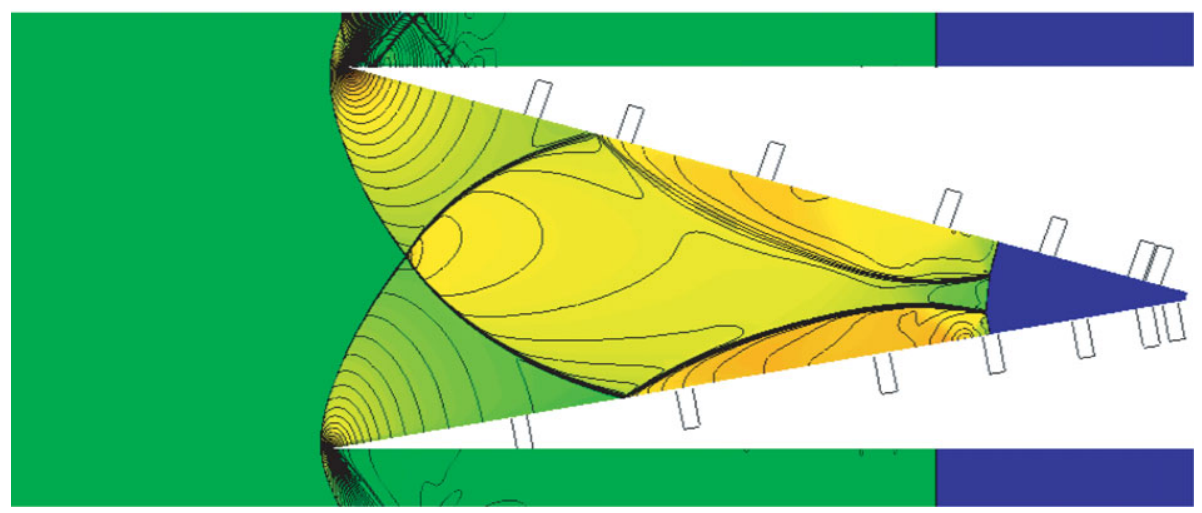

(b) $t=301 \mu \mathrm{s}$

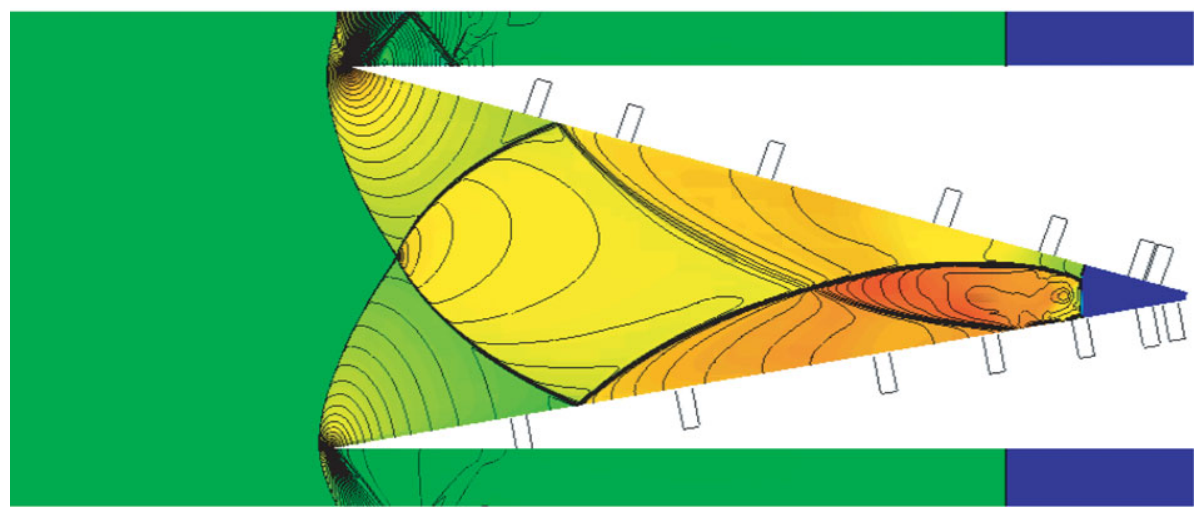

(c) $t=326 \mu \mathrm{s}$

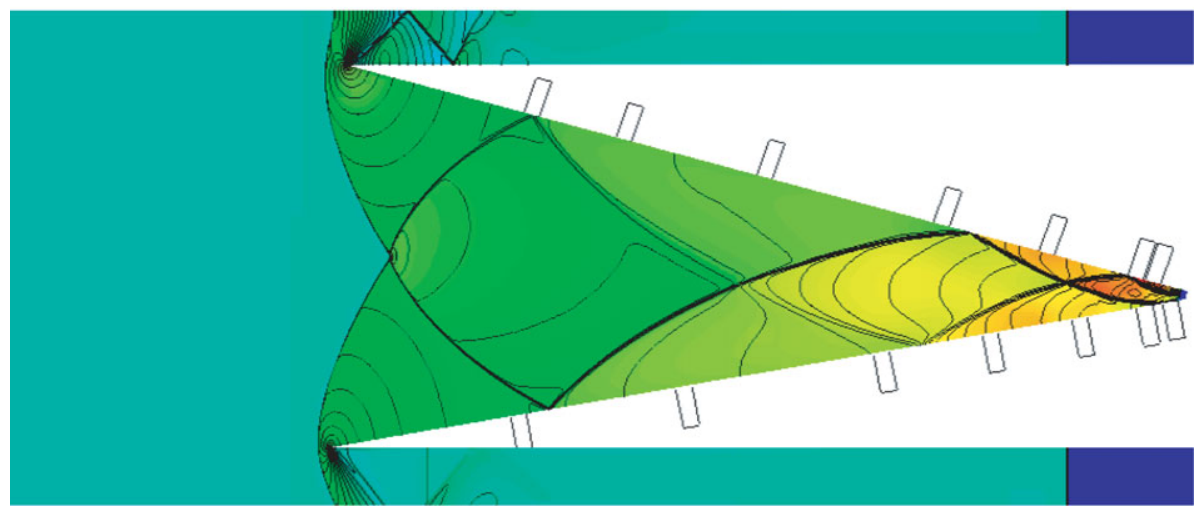

FIGURE 18. Numerical visualization of Run $71, M_{i}=2.46$, as incident shocks hit transducers L4 $(t=273 \mu \mathrm{s})$, L5 $(t=301 \mu \mathrm{s})$ and L7. $(t=326 \mu \mathrm{s})$.

and $\gamma$ is the ratio of specific heats. The area of the cylindrical shock is given by $A=2 \pi r$, so, given the initial Mach number $M_{0}$ and the radius $A_{0}=2 \pi r_{0},(6.1)$ gives the radius as a function of Mach number as the shock converges. 
(a) Reflected shocks overlap

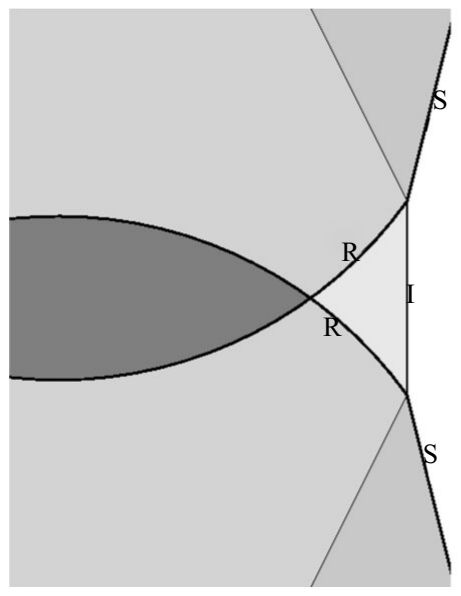

(b) Shock strengthened

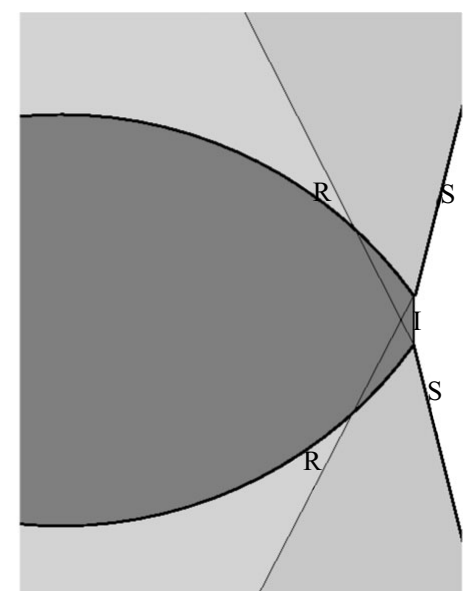

FiguRE 19. Diagrams showing the local flow conditions just prior and after the triple-point crossing. Here, $\boldsymbol{I}$ is the incident shock, $\boldsymbol{R}$ labels the individual Mach Reflections and $\boldsymbol{S}$ indicates the individual Mach stems.

(a) Run $69, M_{i}=1.49$

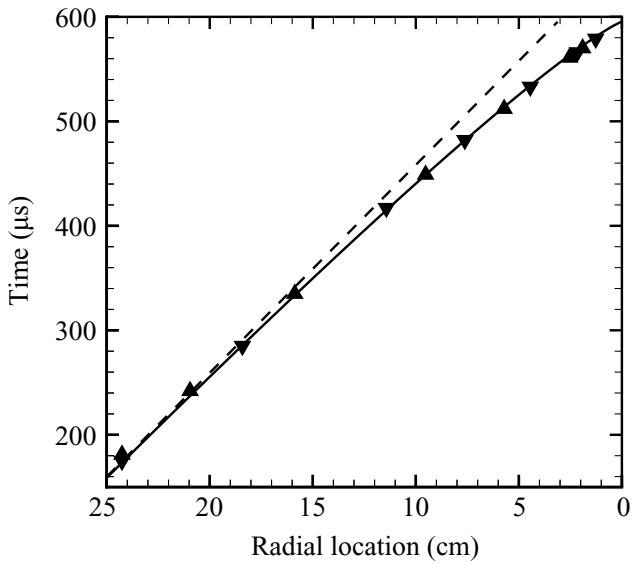

(b) Run $71, M_{i}=2.43$

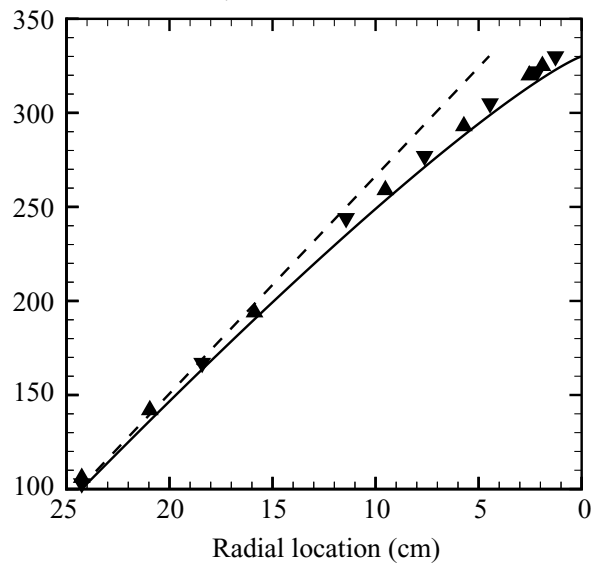

FIGURE 20. The shock location as a function of time. The solid line represents the shock dynamics theory for a circular shock and the dashed line is a simple planar shock. The triangles and inverted triangles correspond to the data taken from the upper plate and lower plate transducers, respectively.

Figure 20 shows the results of a comparison of the planar and convergent cases with experimental results from both cases N1.5 (left) and N2.5 (right). The dashed line indicates the timing of a planar shock with the same initial strength as the wave entering the wedge. The solid line indicates the excellent agreement between the shock dynamics solution for a cylindrically converging shock.

Interestingly, the N1.5 case experimental results fall almost on top of the cylindrical wave. The N2.5 case, however, deviates from the cylindrical case, with focusing that lags the cylindrical shock in arrival time.

To obtain insight into the differences in focusing, the computations of the two cases were compared with the shock dynamics solution. The instantaneous Mach number 
(a) $M_{i}=1.49$

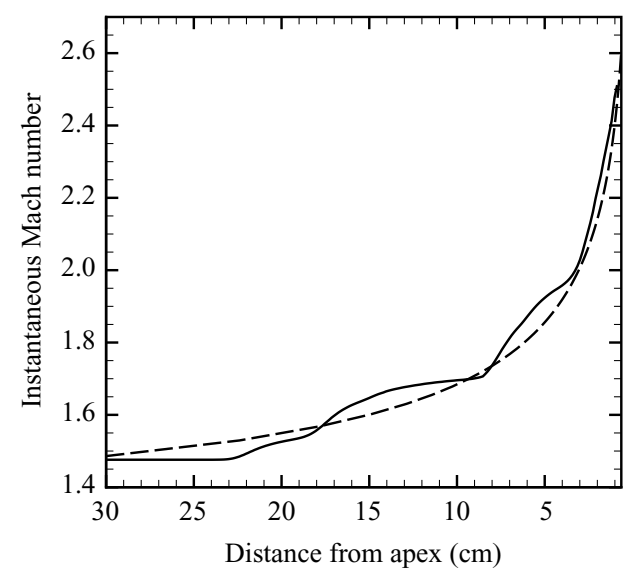

(b) $M_{i}=2.46$

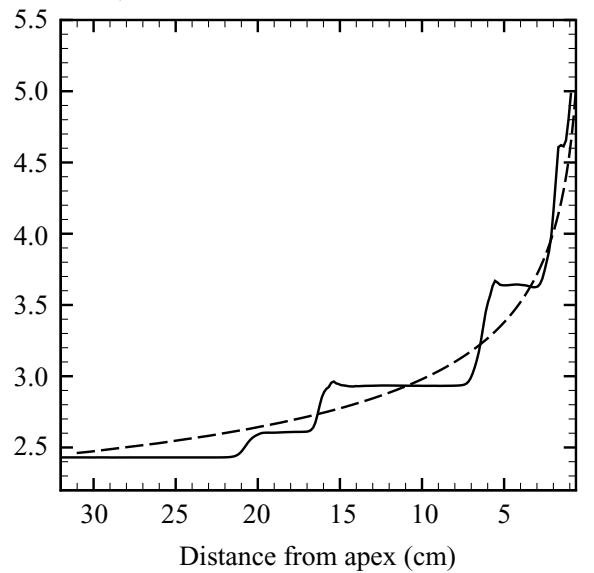

FIGURE 21. The shock Mach number measured on the horizontal as a function of distance. The solid line represents the computed instantaneous Mach number and the dashed line is given by the shock dynamics theory.

of the incoming shock was recorded as a function of position on the horizontal (emanating from the wedge apex) and is plotted in figure 21. There is an obvious distinction between the two cases; the N1.5 case has a number of distinct, but smooth, increases in shock Mach number, while in the N2.5 case, the increase in shock Mach number is made in a stepwise fashion. This difference in behaviour is consistent with the previous discussion of both the triple points and the reflected wave pressure traces; the distributed nature of the N1.5 reflections results in much smoother focusing, while the steeper and less distributed N2.5 reflections result in the stepwise focusing at the higher incoming shock Mach number.

\section{Real-gas effects in carbon dioxide flows}

It has been demonstrated in the preceding sections that the lower incident Mach number $\left(M_{i}=1.5\right)$ experiment produced smoother focusing, and this is attributed to the nature of the reflected shocks. Additional converging planar shock experiments were performed utilizing the same Mach numbers, experimental apparatus and diagnostic system, but with $\mathrm{CO}_{2}$ in place of $\mathrm{N}_{2}$ (see table 1). In contrast to $\mathrm{N}_{2}$, which may be treated as an ideal gas for the experiments, $\mathrm{CO}_{2}$ exhibits a real-gas behaviour in the form of relatively slow vibrational relaxation under pressures and temperatures similar to those in the $\mathrm{N}_{2}$ experiments. The introduction of significant relaxation effects places this experiment in a regime beyond the immediate scope of the local triple-point theory, but these same real-gas effects ensure that the reflected waves will include an enhanced distributed region. Table 6 summarizes the flow conditions for these experiments.

The results reported here constitute a brief investigation into focusing with real-gas effects in the form of vibrational relaxation. As will be seen, the results are very similar to those seen in the preceding sections, although the reflected waves produce somewhat smoother transducer pressure rises and overall focusing. 


\begin{tabular}{|c|c|c|c|c|c|c|c|}
\hline \multirow[b]{2}{*}{ Run } & \multicolumn{2}{|c|}{ Driver } & \multirow{2}{*}{$\begin{array}{c}\text { Test section } \\
\quad p(\mathrm{kPa})\end{array}$} & \multicolumn{4}{|c|}{ Measured parameters } \\
\hline & Gas & $p(\mathrm{kPa})$ & & $M_{i}$ & $\delta_{\mathrm{t}}(\mu \mathrm{s})$ & $t_{\text {picture }}(\mu \mathrm{s})$ & $T\left({ }^{\circ} \mathrm{C}\right)$ \\
\hline 73 & $\mathrm{He}+20 \%$ air & 100 & 19.7 & 1.51 & 319.0 & 199.5 & 24.0 \\
\hline 74 & $\mathrm{He}+20 \%$ air & 103 & 19.7 & 1.52 & 316.5 & 1400 & 24.3 \\
\hline 75 & $\mathrm{He}+5 \%$ air & 85 & 2.40 & 2.55 & 189.0 & 151.2 & 24.3 \\
\hline 77 & $\mathrm{He}+5 \%$ air & 83 & 2.41 & 2.50 & 192.7 & 1150 & 24.1 \\
\hline
\end{tabular}

TABLE 6. Run conditions for individual $\mathrm{CO}_{2}$ runs.

\subsection{Simulation of $\mathrm{CO}_{2}$ experiments}

As expected, assuming a constant ratio of specific heats, $\gamma$ for $\mathrm{CO}_{2}$, or using a temperature-dependent $\gamma(T)$ in the simulations, failed to produce good agreement with experiment. It was found necessary to model the effect of the additional degrees of freedom (i.e. molecular vibrational modes) by modifying the form of internal energy density $e$ and the related equation of state. When discussing departure from the ideal-gas behaviour, it is simplest to represent $e$ as the sum of the translation and rotation energy density $e^{t+r}$ with vibrational energy density $e^{v i b}$ to be modelled. This vibrational energy density is in turn modelled for $\mathrm{CO}_{2}$ by a decomposition into the four basic vibrational modes

$$
e=e^{t+r}+\sum_{m=1}^{4} e_{m}^{v i b} .
$$

Here, as $\mathrm{CO}_{2}$ is a linear molecule, the translation and rotation energy may be written as $e^{t+r}=5 R T / 2$, where $R$ is the gas constant for $\mathrm{CO}_{2}$ and $T$ is the translationalrotational temperature related to the pressure by $p=\rho R T$. The vibrational modes are taken to be the $m=1$ symmetric stretching mode, the $m=2$ and $m=3$ bending modes, and the $m=4$ asymmetric stretching mode. The quantum harmonic oscillator theory predicts (Vincenti \& Kruger 1965; Zel'dovich \& Raizer 2002) that each of the vibrational modes, when in equilibrium at a temperature $T$, will have an energy density given by

$$
e_{m}^{*}(T)=R \frac{\theta_{m}}{\exp \left(\theta_{m} / T\right)-1},
$$

with, for $\mathrm{CO}_{2}$, the characteristic temperatures $\theta_{1}=1920 \mathrm{~K}, \theta_{2}=\theta_{3}=960 \mathrm{~K}$ and $\theta_{4}=3380 \mathrm{~K}$.

To model the relaxation of the different vibrational modes, the standard Euler equations are augmented by the addition of four rate equations in the advection form

$$
\frac{\partial \rho e_{m}^{v i b}}{\partial t}+\frac{\partial \rho e_{m}^{v i b} u_{j}}{\partial x_{j}}=\rho \frac{e_{m}^{*}(T)-e_{m}^{v i b}}{\tau_{m}(T, p)},
$$

where the characteristic times, $\tau_{m}$, are given by the Teller-Landau theory (for details, see Zel'dovich \& Raizer 2002),

$$
\ln \left(\frac{\tau_{m} p}{C_{m}}\right)=\left(\frac{T_{o}}{T}\right)^{1 / 3},
$$

with the dimensional constants $C_{1}=C_{4}=2.2 \times 10^{-7} \mathrm{sec} \cdot \mathrm{atm}, C_{2}=C_{3}=C_{1} / 10$ and $T_{o}=(37)^{3} K$. 
(a) Run $73, M_{i}=1.5$, schlieren

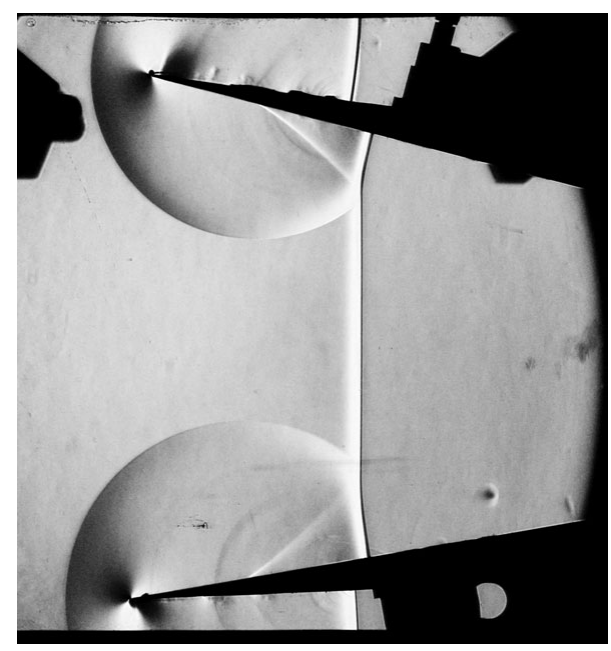

(c) Run $75, M_{i}=2.55$, schlieren

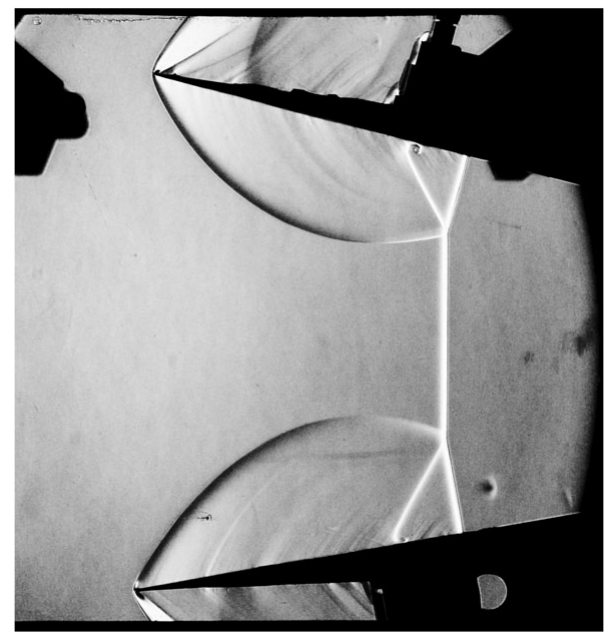

(b) Run 73 pressure contours

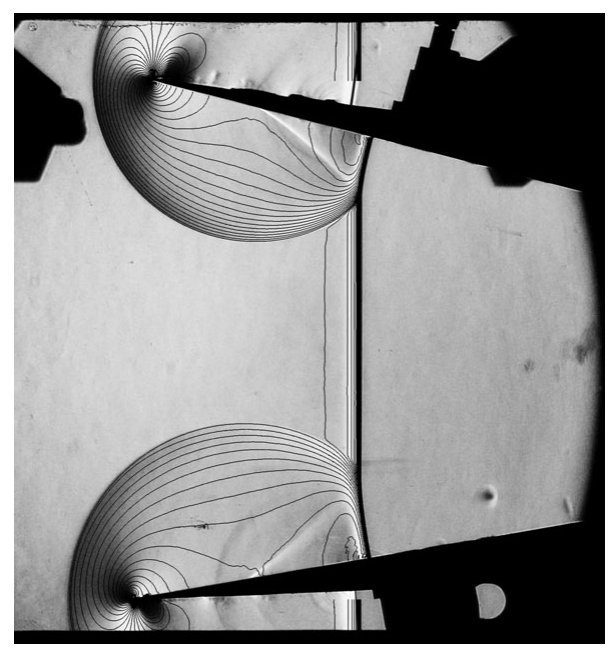

(d) Run 75 pressure contours

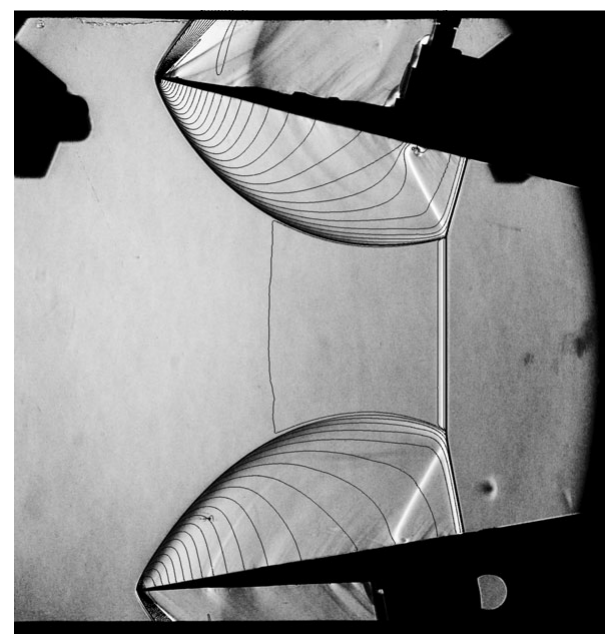

FIGURE 22. Comparison of schlieren and the simulation of shocks in $\mathrm{CO}_{2}$. The evidence of the relaxation zones is clear in both the schlieren and the pressure contours. Weak waves apparently originating on the wedge surfaces may be due to the roughness and limitations of the gaskets between the windows and the wedge.

\subsection{Results and discussion of $\mathrm{CO}_{2}$ experiments and simulations}

Figure 22(a) shows a C1.5 case (Run 73), with a shock Mach number of 1.51 in $\mathrm{CO}_{2}$ and figure $22(b)$ includes the computed pressure contours. While the overall flow configuration looks similar to the N1.5 case (figure 3), the real-gas effects are noticeable. The incoming shock (in the centre of the picture, between the two Mach reflections) has a clear relaxation zone behind the leading jump, as expected for a shock in $\mathrm{CO}_{2}$ (Johannesen et al. 1962). Relaxation zones are also evident behind the Mach stems and the reflected waves. Although the relaxing flow makes for a more difficult diagnosis, the curvature of the shock intersection region indicates that this is also a distributed reflection. The image suggests that the flow is largely similar to that 
(a) $M_{i}=1.51$

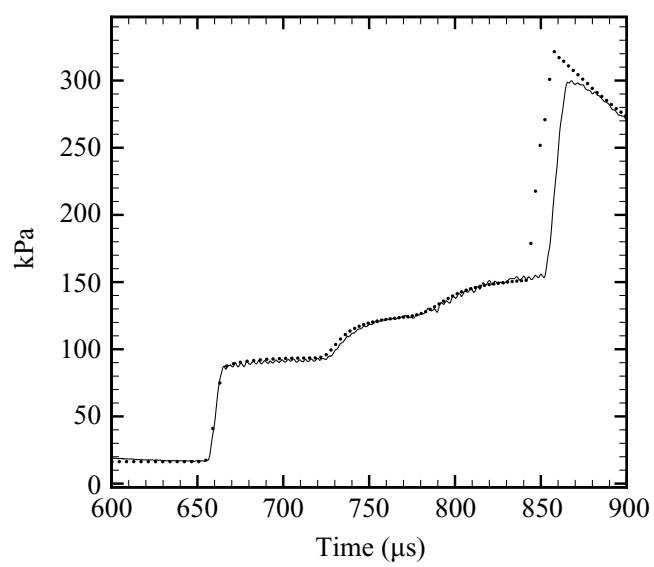

(b) $M_{i}=2.55$

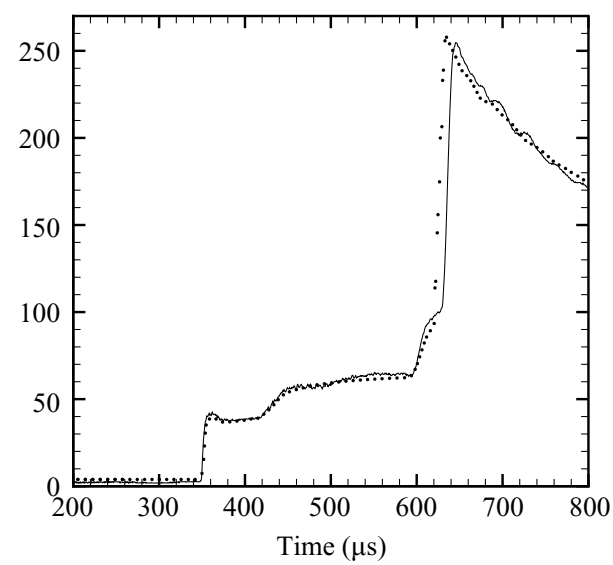

Figure 23. $M_{i}=1.51$ and $M_{i}=2.55$ in $\mathrm{CO}_{2}$ : A comparison of the simulated pressure traces (circle) with the experiment (line) for the fourth transducer on the bottom plate. As with the $\mathrm{N}_{2}$ experiments, leakage at the hinge produces a significant offset in the time of the returning shock.

of the N1.5 case, although slightly modified by relaxation, and this in turn suggests that the distributed nature of the reflection is not particularly sensitive to the presence of a weak shock leading the reflection, since any such weak shock would be fully dispersed at such a low shock Mach number. A comparison of the pressure contours from the Mach 1.5 shock in $\mathrm{CO}_{2}$ with the corresponding contours for the $\mathrm{N}_{2}$ case shows that the introduction of real-gas effects does little to alter the structures of the reflected shocks, although the pressure rise in the following reflected wavefront is smoother in the $\mathrm{CO}_{2}$ case. One can also see that the distribution of pressure between the slipline and wedge plates differs; in the $\mathrm{N}_{2}$ simulation, a pressure maximum can be found at the Mach stem, but the relaxation effects move that pressure maximum away from the Mach stem in $\mathrm{CO}_{2}$.

Figures 22(c) and 22(d) may be compared with figures 6 and 14(b), respectively. Again, the flows are similar, but the relaxation zones at the leading shocks are evident in the $\mathrm{CO}_{2}$ image and this results in a smoother pressure rise following the shocks. The pressure contours within the reflected wave in the area near the triple point are largely parallel to the reflected wavefront in figure $22(d)$ in contrast to those in the $\mathrm{N}_{2}$ case in figure $14(b)$, where the contours appear to terminate in the reflected wavefront.

In figure 23, one sees that the agreement with computation prior to the returning shock is quite good. One will notice that the pressure rises are much smoother in the $\mathrm{CO}_{2}$ experiments than that in the corresponding $\mathrm{N}_{2}$ pressure histories (figure 8). This is probably a function of the relaxation zones at the front of the reflected waves.

While the authors are not aware of a simple formula for the strength of a converging cylindrical shock in the presence of real-gas effects, the results of the Mach number along the horizontal from the simulation of the $\mathrm{CO}_{2}$ experiments may still be compared with a shock dynamics prediction for a cylindrical shock using an ideal-gas approximation to $\mathrm{CO}_{2}$ by assuming a constant $\gamma=1.29$. As seen in the $\mathrm{N}_{2}$ investigation (figure 21), the lower incident Mach number shock results in a smoother focusing (figure 24). It is interesting that the instantaneous Mach number in figure 24( $a)$ oscillates about the cylindrical shock prediction. This is a rather subtle 
(a) $M_{i}=1.51$

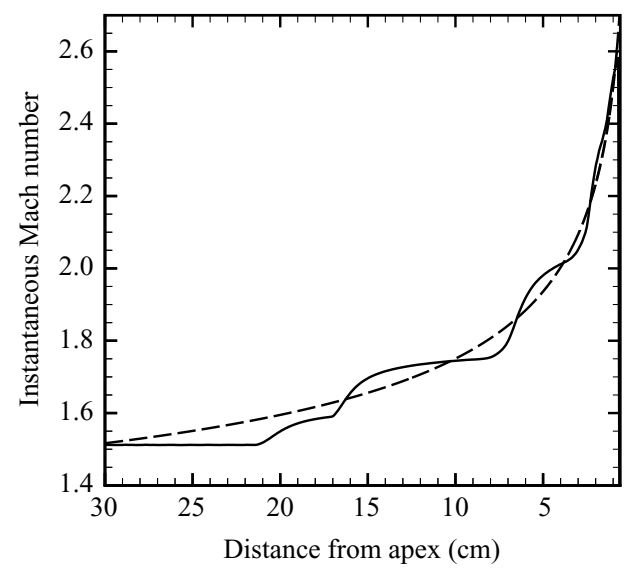

(b) $M_{i}=2.55$

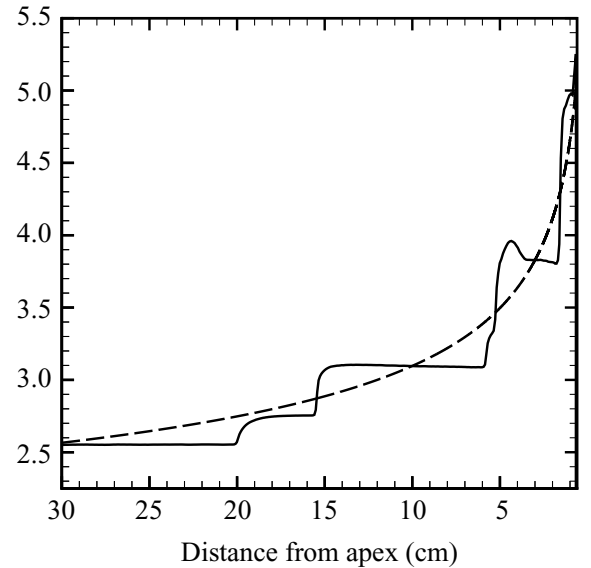

FIGURE 24. The shock Mach number measured on the horizontal as a function of distance. The solid line represents the computed instantaneous Mach number and the dashed line is given by the shock dynamics theory for an ideal gas with $\gamma=1.29$.

difference from the $\mathrm{N}_{2}$ case (figure 21) but consistent with the idea that the enhanced smoothness of the reflected waves owing to relaxation effects results in a shock closer to an arc of a cylindrical shock. A more important consequence of the real-gas effects for this flow is the significant difference between the behaviour of the reflected outgoing wave structures between $\mathrm{N}_{2}$ and $\mathrm{CO}_{2}$. This is discussed in Appendix $\mathrm{A}$, as it is not part of the shock focusing that precedes it.

\section{Conclusions}

Focusing of a planar shock in a two-dimensional linearly converging geometry was investigated with experiments and numerical simulations. The simulations were validated by a comparison with experimental results and subsequently used to augment and facilitate a detailed examination of the physics of these flows. Two incoming shock strengths were selected: one where the initial Mach reflections are within the distributed Mach reflection regime, where most of the compression is associated with the curved Mach stem and not with a leading shock, and the other within the compact Mach reflection regime where most of the compression is associated with the shock leading the reflection. The current investigation is an attempt to understand focusing in the flows where distributed reflections arise, and how they differ from flows with compact reflections, where the focusing, dominated by repeated reflections of triple points off the wedge walls, is better understood.

The structure of the initial reflections (before the interaction between the two reflections) corresponds well to previous investigations. The flows in the N1.5 case behave as one would expect from the Colella \& Henderson (1990) results, with a significant portion of the reflected wave in the compression fan following the leading edge. The N2.5 case shows nearly straight Mach stems with the reflected wave primarily consisting of a leading shock, quite close to a canonical Mach reflection.

A surprising result is that the extended structure of the reflected waves persists even after multiple interactions with the opposing wedge plates and attendant increases in the pressure. While the distributed reflected waves might be expected to steepen to shocks after the self-similar regime is broken, pressure traces from both experiment 
and simulation indicate that the reflected wave structures remain distributed and that differences in the initial reflection structure remain clearly visible in the images produced by simulation, even for the later stages of the experiments.

The timing of the incoming shocks was compared with both unfocused planar shocks and theoretical predictions for converging cylindrical shocks. The N1.5 case is found to match the corresponding cylindrical shock well, while the N2.5 case lags its cylindrical counterpart. The differences between these cases are illuminated by comparisons of the shock Mach number along the horizontal axis. The numerical simulations show that the N1.5 case is a closer match with its well-distributed smooth increases of the shock Mach number, while the N2.5 case consists of stepwise increases of Mach number caused by the compact reflected waves.

The nature of the focusing in a two-dimensional converging geometry is then found to depend on the structure of the reflected waves early in the flow; distributed reflections produce much smoother focusing that better approximates a circular cylindrical shock, while the sharper waves from compact reflections outside that regime yield a poorer approximation to smooth focusing.

Subtle changes in the flow fields were observed when using $\mathrm{CO}_{2}$ in place of $\mathrm{N}_{2}$. The form of the initial triple-point regions is quite similar in both gases at comparable Mach numbers. This may be understood by observing that near the front of the shock, and hence the triple points and Mach stem, the vibrational modes in $\mathrm{CO}_{2}$ are not excited and the gas behaves locally as an ideal gas with $\gamma=1.4$, as in $\mathrm{N}_{2}$. The relaxation zone is most prominent in the reflected waves, where the transfer of energy to the vibrational modes produces a dispersed reflected wave in the C1.5 case. This can be clearly seen in pressure overlays for the early stages of the flow and in the pressure traces for the entire experiment. (This difference is more pronounced in the post-focusing outgoing wave system discussed in Appendix A.)

We would like to acknowledge the code development and contributions undertaken as part of the Caltech DOE ASC Alliance Program by R. Deiterding, documented above, on which the numerical simulations relied, the early exploratory discussions and investigations in collaboration with R. Samtaney, the preliminary experimental work by A. Lam, and the mechanical engineering and design assistance of G. Kaztenstein and B. Valiferdowsi. This work was supported by the Advanced Simulation and Computing (ASC) Program under subcontract no. B341492 of DOE contract W7405-ENG-48.

\section{Appendix A. Returning shock propagation into subsonic flow}

While the main text of the paper concentrates on shock focusing, pressure data and schlieren images were also taken for the flow after the shock reached the apex of the wedge. This appendix discusses these results and the differences between the experiments and computations in the flow behind the returning shock.

After the outgoing shock passes a transducer, no further sign of the reflected waves is seen. In both the experimental and numerically simulated pressure traces, the outgoing shock appears as a strong spike followed by a long period of smooth decay. The schlieren images recorded as the shock nears the wedge exit illustrate this.

Figure 25(a) shows the outbound shock of Run 69, initially a Mach 1.48 shock. To the left, the reflected shocks that have bounced off both wedge plates are drifting 
(a) Run $69, M_{i}=1.48$

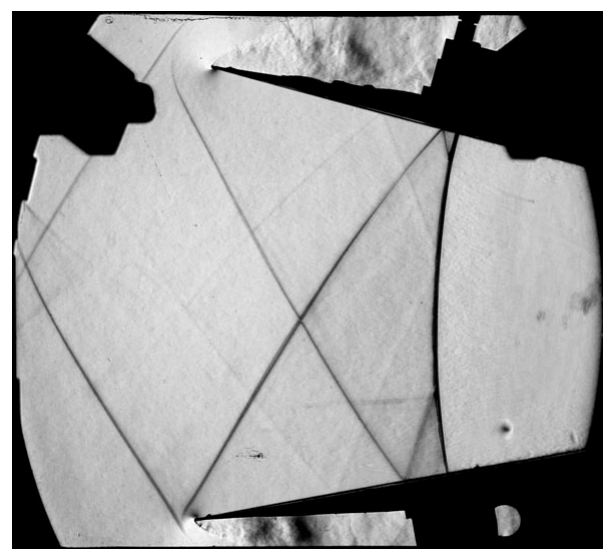

(b) Run $71, M_{i}=2.46$

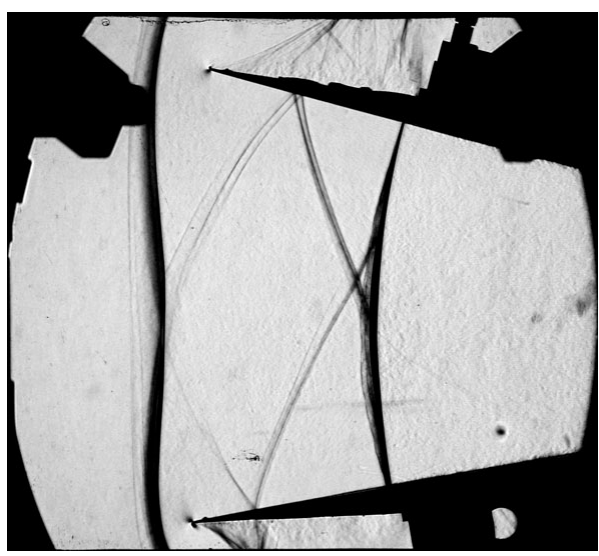

FIGURE 25. Outgoing shock in $\mathrm{N}_{2}$ develops as an expanding cylindrical shock.

upstream towards the shock tube. The reflected shock has coalesced into a nearly cylindrical expanding shock and is about to exit the wedge.

Figure 25(b) shows the outbound shock of Run 71, initially a Mach 2.46 shock. To the left, the reflected waves have built up into a single wave standing just off the wedge tips. The reflected shock here shows a significantly larger amount of three-dimensionality than in the N1.5 case.

Unlike the agreement found between experiment and simulation in the incident phase of the runs, the returning-shock location predicted by the simulation always leads the actual shock in the experiment, as mentioned in the discussion of the pressure traces. While not shown here, during the outgoing phase, the locations of the waves reflected from the wedge plates are well predicted by the computation. For example, the pressure trace comparisons in figures 13 and 23 show good agreement (in both $\mathrm{N}_{2}$ and $\mathrm{CO}_{2}$ ) until the returning shock arrives. This suggests that the difference between the simulation and the experiment is concentrated at the apex of the wedge, and more specifically at the time when the shock focuses in the neighbourhood of the apex.

Figure 26(a) shows both experimental and computational results in an $(r, t)$ diagram of the returning shock. As indicated, the computed shock leads the experimental shock. Figure 26(b) shows the differential timing of the shock arrivals. In this figure, the differences can be seen to grow while the shock is deep in the wedge, but as the shock exits (and the pressure behind the shock drops) the difference reaches a steady state.

The experimental apparatus was designed to allow easily adjustable wedge plate angles with a precision hinge. However, the implementation does not safeguard against small leakage effects after reflection, especially during the short time interval when the hinge assembly is exposed to the high pressure shortly behind the reflected wave. This would also explain why the lag ceases to grow as the shock exits; the pressure differential decreases as the shock leaves the apex.

Figure 27 shows the schlieren images from outgoing flows in $\mathrm{CO}_{2}$; these roughly correspond to the $\mathrm{N}_{2}$ flows in figure 25. The major difference is that the shocks in the $\mathrm{CO}_{2}$ cases show significant real-gas effects. Waves reflected from the wedges in the lower Mach number case are fully dispersed, as expected for shock Mach numbers below $\approx 1.04$. 
(a) Shock arrival times

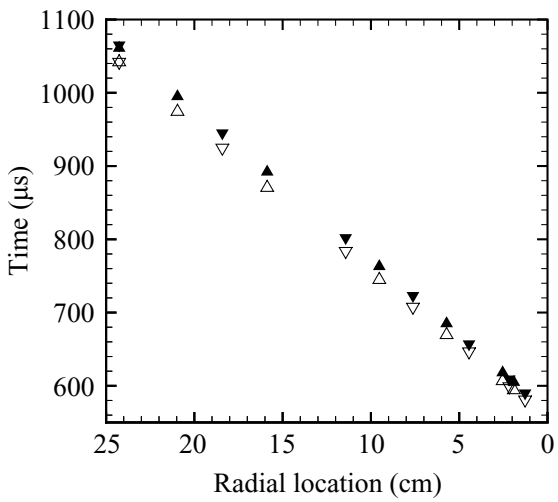

(b) Differential timing

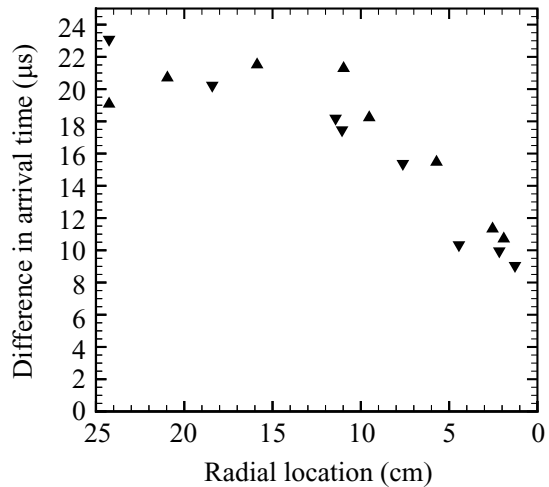

Figure 26. Comparison of Run 69 outgoing shock timings; experimental vs. simulated. In (a) the open symbols are simulation data and the closed symbols are from the experiment, triangles correspond to transducers on the upper plate and inverted triangles indicate the data from the lower plate. In $(b)$ the symbols indicate the difference in shock arrival times for each transducer.

(a) Run $74, M_{i}=1.52$

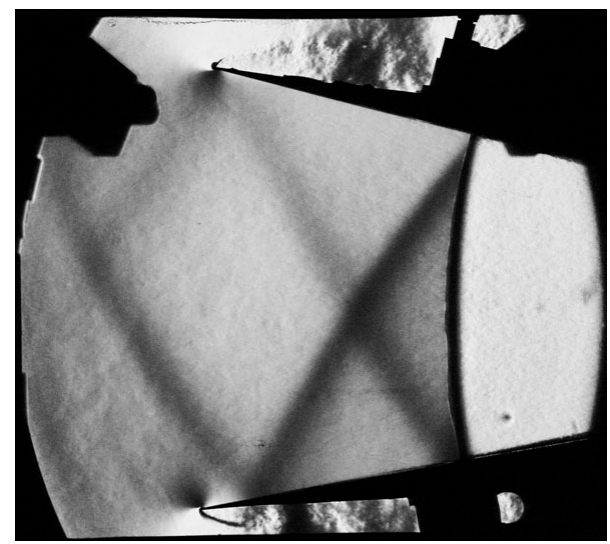

(b) Run $77, M_{i}=2.50$

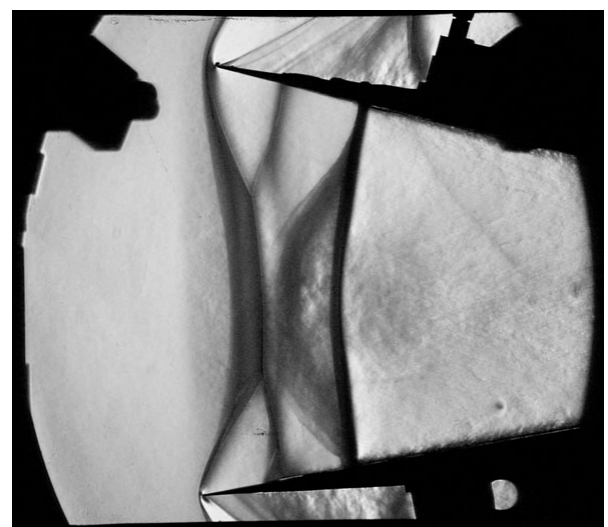

FIGURE 27. Outgoing shock in $\mathrm{CO}_{2}$. Reflected shocks in the lower Mach number case are fully dispersed.

\section{Appendix B. Schlieren system and image processing}

This discussion again places some emphasis on the details that proved important in the comparison between the experiment and the simulation. The images presented in this paper were produced using a compact, Z-fold schlieren system configured with folding in the horizontal plane. The $25 \mathrm{~cm}$ diameter spherical mirrors with a $1.38 \mathrm{~m}$ focal length were used as the focusing mirrors. Folding the light path, two $25 \mathrm{~cm}$ diameter flat mirrors were mounted at $45^{\circ}$ between the focusing mirrors while the camera and light source were mounted $10^{\circ}$ off-axis from the focusing mirrors. The folding mirrors limited the horizontal field of view, while the test section windows limited the vertical field of view.

The light source for the schlieren system was a Model 437B Nanopulser from Xenon Corporation. This source produces a short duration (20 ns) spark and allows time-resolved imaging of the flow. The spark itself was oriented vertically to maximize 
sensitivity in the horizontal direction, and was used as the image light source, without condensers or masks.

On the imaging side of the system, the knife edge was oriented at the vertical focus of the system so that, for small deflections, the signal indicates the horizontal gradient of the index of refraction (i.e. of density). To provide a consistent large deflection cutoff, the knife edge was surrounded by a $10 \mathrm{~mm}$ diameter circular mask centred on the spark image and the knife edge was followed by two $75 \mathrm{~mm}$ diameter achromat lenses (300 $\mathrm{mm}$ and $200 \mathrm{~mm}$ focal lengths). Images were recorded using a Nikon D70s digital SLR with a Nikon AF-S DX Zoon-Nikor ED 18-70 mm f/3.5-4.5G lens, set at a $70 \mathrm{~mm}$ focal length for all images. The images were recorded using the Nikon D70s RAW format and converted to 16 bit TIFF images before further processing; notably the acquired images have a more dynamic range that can be captured with an 8 bit image format.

Optical distortions in the schlieren images were corrected using images of an optical-calibration assembly. The assembly is made from acrylic plastic and consists of a $41 \mathrm{~cm} \times 22 \mathrm{~cm}$ grid plate with a base plate used to position the grid on the test section mid-span plane. The grid plate has a precision machined pattern of $0.5 \mathrm{~mm}$ wide grooves located at $1.27 \mathrm{~mm}$ intervals to a tolerance of $\pm 25.4 \mu \mathrm{m}$. To correct the images for optical distortion, an uncorrected grid picture was loaded into MATLAB. The image of the grid and a mathematically constructed uniform grid were used to create a set of control point pairs (i.e. grid intersections) that were then used to create a third-order polynomial transform with bi-cubic interpolation to convert the distorted image into a corrected image. Finally, the 16 bit images were converted to 8 bit images for viewing, with the choice of brightness and contrast dependent upon the features of interest.

\section{REFERENCES}

Apazidis, N., Lesser, M. B., Tillmark, N. \& Johansson, B. 2002 An experimental and theoretical study of converging polygonal shock waves. Shock Waves 12, 39-58.

BEN-Dor, G. 2006 A state-of-the-knowledge review on pseudo-steady shock-wave reflections and their transition criteria. Shock Waves 15, 277-294.

Ben-Dor, G. 2007 Shock Wave Reflection Phenomena. Springer.

Colella, P. \& Henderson, L. F. 1990 The von Neumann paradox for the diffraction of weak shock-waves. J. Fluid Mech. 213, 71-94.

Deiterding, R., Radovitzky, R., Mauch, S., Noels, L., Cummings, J. \& Meiron, D. 2006 A virtual test facility for the efficient simulation of solid material response under strong shock and detonation wave loading. Engng Comput. 22, 325-347.

Dimotakis, P. E. \& Samtaney, R. 2006 Planar shock cylindrical focusing by a perfect-gas lens. Phys. Fluids 18.

Eliasson, V., Apazidis, N., Tillmark, N. \& Lesser, M. B. 2006 Focusing of strong shocks in an annular shock tube. Shock Waves 15, 205-217.

Evans, A. K. 1996 Instability of converging shock waves and sonoluminescence. Phys. Rev. E 54, 5004-5011.

Gottlieb, S., Shu, C. W. \& Tadmor, E. 2001 Strong stability-preserving high-order time discretization methods. SIAM Rev. 43, 89-112.

Griffith, W. \& Kenney, A. 1957 Fully-dispersed shock waves in carbon dioxide. J. Fluid Mech. 3, $286-288$.

Guderley, G. 1942 Starke kugelige und zylindrische verdichtungsstösse in der nähe des kugelmittelpunktes bzw der zylinderachse. Luftfahrtforschung 19, 302-312.

Henderson, L., Vasilev, E., Ben-Dor, G. \& Elperin, T. 2003 The wall-jetting effect in Mach reflection: theoretical consideration and numerical investigation. J. Fluid Mech. 479, 259-286. 
Hill, D. J. \& Pullin, D. I. 2004 Hybrid tuned centre-difference-WENO method for large eddy simulations in the presence of strong shocks. J. Comput. Phys. 194, 435-450.

Hosseini, S. H. R. \& TAKayama, K. 2005 Experimental study of Richtmyer-Meshkov instability induced by cylindrical shock waves. Phys. Fluids 17.

Inoue, O., Imuta, S., Milton, B. E. \& Takayama, K. 1995 Computational study of shock-wave focusing in a log-spiral duct. Shock Waves 5, 183-188.

Inoue, O., Takahashi, N. \& TaKayama, K. 1993 Shock-wave focusing in a log-spiral duct. AIAA J. 31, 1150-1152.

Johannesen, N., Zienkiewicz, H., Blythe, P. \& Gerrard, J. 1962 Experimental and theoretical analysis of vibrational relaxation regions in carbon dioxide. J. Fluid Mech. 13, 213-224.

Kumar, S., Hornung, H. \& Sturtevant, B. 2003 Growth of shocked gaseous interfaces in a conical geometry. Phys. Fluids 15, 3194-3208.

Liepmann, H. W., Coles, D., Roshko, A. \& Sturtevant, B. 1962 17-inch diameter shock tube for studies in rarefied gas dynamics. Rev. Sci. Instrum. 33, 625-631.

Olim, M. \& Dewey, J. 1992 A revised three-shock solution for the Mach reflection of weak shocks. Shock Waves 2, 167-176.

Pantano, C., Deiterding, R., Hill, D. J. \& Pullin, D. I. 2007 A low numerical dissipation patchbased adaptive mesh refinement method for large-eddy simulation of compressible flows. J. Comput. Phys. 221, 63-87.

Perry, R. W. \& Kantrowitz, A. 1951 The production and stability of converging shock waves. J. Appl. Phys. 22, 878-886.

SANDEMAN, R. J. 2000 A simple physical theory of weak mach reflection ever plane surfaces. Shock Waves 10, 103-112.

Sasoh, A. \& Takayama, K. 1994 Characterization of disturbance propagation in weak shock-wave reflections. J. Fluid Mech. 277, 331-345.

Schwendeman, D. W. \& Whitham, G. B. 1987 On converging shock-waves. Proc. R. Soc. London A-Math. Phys. Engng Sci. 413, 297-311.

Setchell, R. E., Storm, E. \& Sturtevant, B. 1972 Investigation of shock strengthening in a conical convergent channel. J. Fluid Mech. 56, 505-522.

Skews, B. W. \& Ashworth, J. T. 2005 The physical nature of weak shock wave reflection. J. Fluid Mech. 542, 105-114.

Takayama, K., Kleine, H. \& Gronig, H. 1987 An experimental investigation of the stability of converging cylindrical shock waves in air. Exp. Fluids 5, 315-322.

Vasilev, E., Ben-Dor, G., Elperin, T. \& Henderson, L. 2004 The wall-jetting effect in Mach reflection: Navier-Stokes simulations. J. Fluid Mech. 511, 363-379.

Vasilev, E. I., Elperin, T. \& Ben-Dor, G. 2008 Analytical reconsideration of the von Neumann paradox in the reflection of a shock wave over a wedge. Phys. Fluids 20 (4), 046101.

Vincenti, W. \& Kruger, C. 1965 Introduction to Physical Gas Dynamics. Wiley.

Watanabe, M. \& TAKAYAma, K. 1992 Stability of converging cylindrical shock-waves. JSME Intl J. II-Fluid Engng Heat 35, 218-227.

Whitham, G. B. 1957 A new approach to problems of shock dynamics. 1. 2-dimensional problems. J. Fluid Mech. 2, 145-171.

Zel'dovich, Y. B. \& Raizer, Y. P. 2002 Physics of Shock Waves and High-Temperature Hydrodynamic Phenomena. Dover. 